
TID-4500, UC-32

Muthematios and Computers

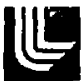 \\ LAMFENCE LNEPMOPE LABOFATOFY

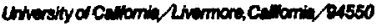

\section{UCRL-S1715 \\ MAGNETOHYDRODYNAMICS OF HEMP}

\author{
Mark L. Wilkins
}

MS. date: March 1973

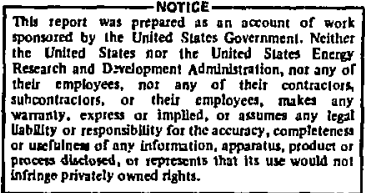

Thls tepott was prepared as in gccount of wotk sponsared by the United States Governmenl. Neither The Unifed States nor the United Sutes Energy suhcontmclors, of their enployees, mikes any wamaniy, express or implied, or asumes any iegalt LabDity of Itsponsiblitty for the accurdey, completeness or unerinca of any information, apparatus, ptoduct of inftrage privately owned tights. 


\section{Contents}

Nolation . . . . . . . . . . . . . . . . . . . . . . , . . . . . . v

Units. . . . . . . . . . . . . . . . . . . . . . . . . . . . . . . . . vi

Abstract , . . , . . . . . . . . . . . . , . . . . . . . . . . 1

Eackground . . . . . . . . . . . . . . . . . . . . . . . . . . . . . 1

Method . . . . . . . . . . . . . . . . . . . . . . . . . . . . . . . , . I

Fundamental Equations of Magnetohydrodynamics . . . . . . . . . . . . . . . . . . . . . 4

Equation of Motion . . . . . . . . . . . . . . . . . . . . . . . . . . . . 4

Electromagnetic Field Equations . . . . . . . . . . . . . . . . . . . . . . 4 t

Energy Equation. . . . . . . . . . . . . . . . . . . . . . . . . . . . . 5

Continuity Equation . . . . . . . . . . . . . . . . . . . . . . . . . . 5

Constitutive Relations . . . . . . . . . . . . . . . . . . . . . . . . . . . 5

Difference Equations for Magnetohydrodynamics . . . . . . . . . . . . . . . . . . . . . . 6

Equations of Motion , . , . . , . , , . . . . . . . . . . . . , . . . . . 6

Magnetic Diffusion . . . . . . . . . . . . . . . . . . . . . . . . . . . 6

Energy Equations . . . . . . . . . . . . . . . . . . . . . . . . . . . . 8

Temperature Diffusion. . . . . . . . . . . . . . . . . . . . . . . 9

Space-Centering of Parameters at Interfaces of Consecutive Zones . . . . . . . . . . . . . . . . 8

Ohmic Heating . . . . . . . . . . . . . . . . . . . . . . . . . . . 9

Continuity Equation . . . . . . . . . . . . . . . . . . . . . . . . . . 10

Time-Step Control . . . . . . . . . . . . . . . . . . . . . . . . . . . . 10

Eoundary Conditions . . . , . . . . . . . . . . . . . . . . . . . . . 10

Sliding Interf́aces . . . . . . . . . . . . . . . . . . . . . . . . . . . . . 11

Check Problems . . . . . . . . . . . . . . . . . . . . . . . . . . . . 12

Acknowledgments . . . . . . . . . . . . . . . . . . . . . . . . . . . . 14

APPENDICES

A. Magnetic Flux Calculation . . . . . . . . . . . , . . . . . . . . . . , , . . 18

Calculation Scheme for k-Sweep. . . . . . . . . . . . . . . . . . . . . . . 18

Calculation Scheme for j-Sweep . . . . . . . . . . . . . . . . . . . . . . . . 25

B. Thermal Diffusion Calculation . . . . . . . . . . . . . . . . . . . . . . . . 33

Calculation Scheme for k-Sweep . . . . . . . . . . . . . . . . . . . . . . 33

Calculation Scheme for j-Sweep . . . . . . . . . . . . . . . . . . . . . . . 41

c. Backward Substitution Method for Solving Linesr Equations . . . . . . . . . . . . . . 49 


\section{List of Illustrations}

1. Integraticn stheme . . . . . . . . . . . . . . . . . . . . . . . . . . 3

2. Gutd for salutulation $\nabla \times \overrightarrow{\mathbf{H}}$. . . . . . . . . . . . . . . . . . . . . . . . . . . . 3

3. Grid for tulculation of $\nabla \times \nabla \times \vec{H}$. . . . . . . . . . . . . . . . . . . . . . . . . 4

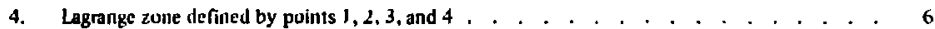

5 Grid showing culculation of flux change in the k-direction . . . . . . . . . . . . . 7

6. Grid showing caltulation of flux change in the j-direction . . . . . . . . . . . . . . . 7

7. Nodis and zanes associated with zone 5 . . . . . . . . . . . . . . . . . . . . 9

8. Grid for culculating diffusion across a silding inter face . . . . . . . . . . . . . . . . 11

9. Application of slide lines in two directions . . . . . . . . . . . . . . . . . . 12

10. Cascuitutional grid to test $\mathbf{T}^{\mathbf{4}}$ diffusion . . . . . . . . . . . . . . . . . . . . . . . . 13

11. Conosisisn of the Ginite difference caleulation with a theoretical calculation for the diffusion

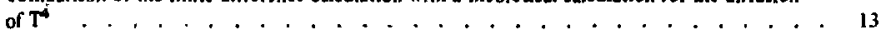

12. Calculation of thermal diffusion in two space dimensions and time . . . . . . . . . . . 15

13. Calculate $d$ temperature contours at $t=1.02 \mu$ s for three calculational grids . . . . . . . 16

A-1. Calculational schene furk-sweep . . . . . . . . . . . . . . . . . . . . . 18

A.2. Calculational grid for flux change calculations . . . . . . . . . . . . . . . . . . . . 20

A.3. Grid for ealctiating flux change from contributions in the k-direction . . . . . . . . . . 23

A4. Calculational git for j-sweep . . . . . . . . . . . . . . . . . . . . . . 25

A.5. Calculational grit. . . . . . . . . . . . . . . . . . . . . . . . . . . . . 28

A-6. Grid for talkulutiby thux change in zone 5 from contributions in the j-direction . . . . . . 31

B-1. Calculational schence for k-sweep . . . . . . . . . . . . . . . . . . . . . . . 33

B.2. Calculational grid . . . . . . . . . . . . . . . . . . . . . . . . . . . . 34

B-3. Grid for calculating teirperature diffusion from contributions in the k-direction . . . . . . 37

B4. Calculational grid for j-siveep . . . . . . . . . . . . . . . . . . . . . . . . 41

B-5. Calculstional grid. . . . . . . . . . . . . . . . . . . . . . . . . . . . . . . 43

B-6. Grid for culculsting tempetature flux change in zone 5 from cuntributions in the j-direction . . 46 


\section{Notation}

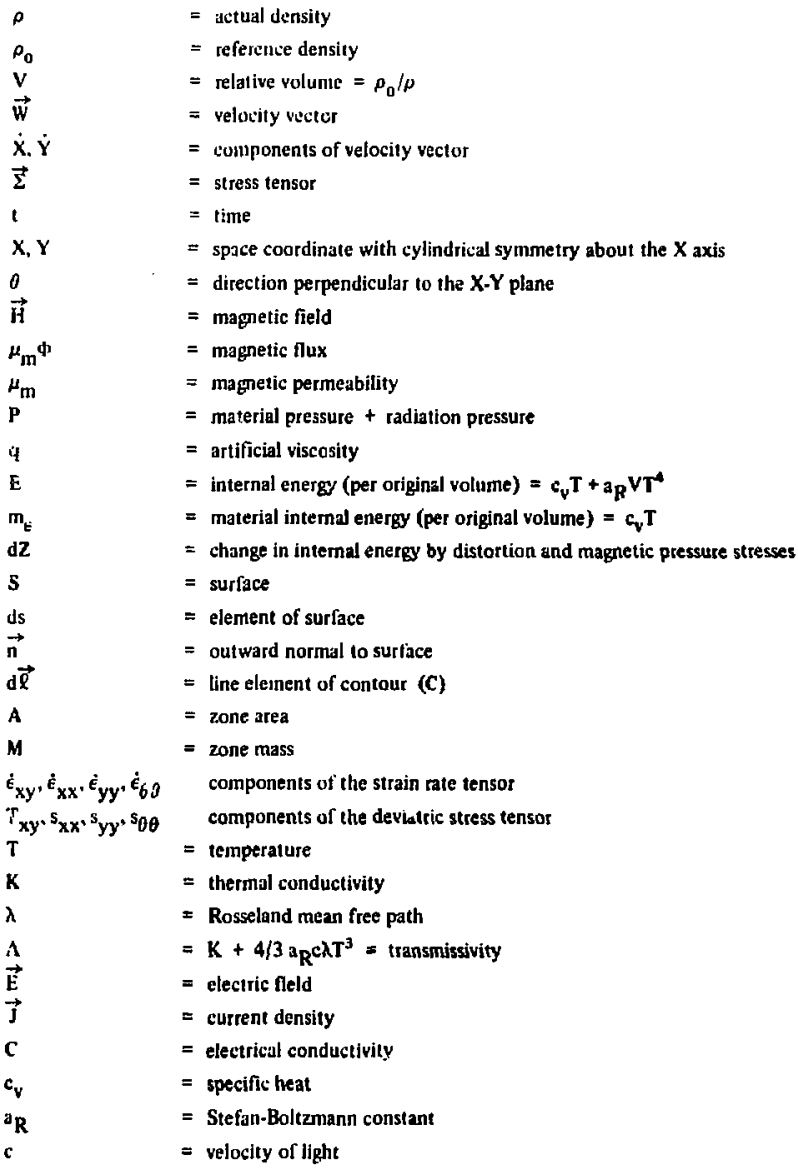




\section{Units}

\begin{tabular}{|c|c|c|c|}
\hline Symbal & Quantity & Units & Sl equivalen: \\
\hline eu & energy unit & $10^{12} \mathrm{ergs}$ & $100 \mathrm{~kJ}$ \\
\hline$\rho$ & densily & $\operatorname{gn} / \mathrm{Lc}$ & $\mathrm{Mg} / \mathrm{m}^{3}$ \\
\hline $\mathbf{v}$ & relative volunie & dimensionless & - \\
\hline E & internal energy per original volu:.te & $\underset{\operatorname{cu}}{\operatorname{cu}} \times \rho^{0}=\frac{\mathrm{cu}}{\mathrm{cnl}^{3}}$ & $\frac{100 \mathrm{GJ}}{\mathrm{m}^{3}}$ \\
\hline 1 & time, $\mu \mathrm{s}$ & $\mu s=10^{\circ} \mathrm{sec}$ & $\mu s$ \\
\hline $\mathrm{X}, \mathrm{Y}$ & space soordinates & cm & $10 \mathrm{~mm}$ \\
\hline$\dot{\mathrm{X}}, \dot{\mathrm{Y}}$ & velocity & $\mathrm{cn} / \mu \mathrm{s}$ & $10 \mathrm{~km} / \mathrm{s}$ \\
\hline $\mathbf{T}$ & Iemperalure & $\mathbf{K}$ & $\mathbf{K}$ \\
\hline $\mathbf{p}$ & pressure (and stress) & $M b=10^{: 2} \frac{\text { dynes }}{\mathrm{ctn}^{2}}$ & $100 \mathrm{GPa}$ \\
\hline $\overrightarrow{\mathbf{H}}$ & magnetic field & $10^{6}$ gauss & $100 \mathrm{~T}$ \\
\hline $\overrightarrow{\mathbf{E}}$ & elestrical field & $\frac{10^{4} \text { volts }}{\mathrm{cm}}$ & $\mathrm{MV} / \mathrm{m}$ \\
\hline $\overrightarrow{\mathbf{j}}$ & current density & $\frac{10^{7} \mathrm{amps}}{\mathrm{cm}^{2}}$ & $100 \mathrm{GA} / \mathrm{m}^{2}$ \\
\hline $\mathbf{C}$ & electrucal conductivity & $\frac{10^{3} \mathrm{mho}}{\mathrm{cm}}$ & $100 \mathrm{kS} / \mathrm{m}$ \\
\hline$\lambda$ & Rosseland mean free path & con & $10 \mathrm{~mm}$ \\
\hline $\mathbf{K}$ & coefficient of thernal conduction & $\frac{e u}{(\mathrm{~cm})\left({ }^{\circ} \mathrm{K}\right)} \cdot \frac{}{(\mu \mathrm{s})}$ & $10 \mathrm{TJ} / \mathrm{m} \cdot \mathrm{K} \cdot \mathrm{s}$ \\
\hline $\mathbf{c}_{\mathbf{v}}$ & specific heat & $\frac{\mathrm{eu} \times \rho^{0}}{(\mathrm{gm})\left({ }^{\circ} \mathrm{K}\right)}=\frac{\mathrm{eu}}{\left({ }^{\circ} K\right)\left(\mathrm{cm}^{3}\right)}$ & $100 \mathrm{GJ} / \mathrm{k} \cdot \mathrm{m}^{3}$ \\
\hline${ }^{\mathbf{a}} \mathbf{R}$ & Stefan-Boltzmann radiation constant & $\frac{756 \times 10^{-27}}{\left(\mathrm{~cm}^{3}\right)\left({ }^{\circ} \overline{\mathrm{K}}^{4}\right)}$ & $756 \mathrm{aJ} / \mathrm{K}^{4} \cdot \mathrm{m}^{3}$ \\
\hline c & velocity of light & $3 \times 10^{4} \frac{\mathrm{cm}}{\mu \mathrm{s}}$ & $390 \mathrm{Mm} / \mathrm{s}$ \\
\hline$\mu_{m}$ & magnetic permeability & 1 & 1 \\
\hline
\end{tabular}




\title{
MAGNETOHYDRODYNAMICS OF HEMP
}

\begin{abstract}
Using the continuum mechanics approach, detais are presented for solving the equations of magnetohydrodynamics in two space dimensions and time. The problem considers cylindrical symmetry, in that only the $\mathrm{H}_{\theta}$ component of a magnetic field is present. $\mathrm{T}_{1}$.

problem is formulated so that the stress contributions resulting from a magnetic field are incorporated into the stress tensor as an elastic-plastic computer program. In addition to the Lorentz force and magnetic diffusion, thermal and radiation diffusion are also treated.
\end{abstract}

\section{Background}

The HEMP code ${ }^{1}$ solves problems in dynamic plasticity in Lagrange coordnates with two space dimensions und cylindrical symmetry. The computer program was originally formulated to include the equations appropriate to magnetohydrodynanics, HEMP being an acronym of the words Hydrodynamic Elastic Nagneto Plastic. Herc we present the inagnetohydrodynamics portion of the code, including thermal and radiation diffusion. Details of the calculational procedure arc given for the case where only the $\overrightarrow{\mathrm{H}}_{\theta}$ component of an applied magnetic field, $\overrightarrow{\mathbf{H}}$, is included. The problem consists of developing a finite difference approximation to the double operator, $\nabla \times \nabla \times \vec{V}$, where $\vec{\nabla}$ is a vector function. The muthematical problem is similar to that for approxinating the double operator, $\nabla \cdot \nabla V$, where $V$ is a scalar function. For the problem at hand, $\vec{V}$ is the magnetic ficld, $\vec{H}$, and $V$ is the temperature, $T$. I is seen from the vector identity:

$$
\begin{aligned}
& \nabla \times \nabla \times \vec{H}=\nabla(\nabla \cdot \vec{H})-\nabla \cdot \nabla \vec{H} \text { that } \\
& \nabla \times \nabla \times \vec{H}=-\nabla \cdot \nabla \vec{H}=-\nabla^{2} \vec{H}, \text { since } \\
& \nabla \cdot \vec{H}=0 .
\end{aligned}
$$

In the calculation of thermal diffusion of the temirara. ture, $T$, a simple change of $\vec{H}$ for $T$ cannot be made in the difference equations. The reason is that in cylindrical coordinates the Luplacian opertion, $\nabla^{2}$, yields a different result when applied to a vector than it does when applied to a scalar quantity.

For the case considered here, where $\overrightarrow{\mathrm{H}}=\overrightarrow{\mathrm{H}}_{\theta}$, the relationship between the Laplacian applied to the vector, $\overrightarrow{\mathrm{H}}_{\theta}$ and to a scaler, $\mathrm{H}_{\theta}$ is:

$$
\nabla^{2} \overrightarrow{\mathbf{H}}_{\theta}=\nabla^{2} \mathbf{H}_{\theta}-\frac{\mathbf{H}_{\theta}}{\mathbf{R}^{2}},
$$

Here $\mathrm{R}$ is the radial space coordinate.

An important considetation in calculating magnetic diffusion or thermal diffusion is the manner in which the constitutive relations are introduced into the difference equations. The constitative relations describing the electrical conductivity in the calculation of magnetic diffusion can be included in a more physical way if the cirl-curl formulation is used. Thus for the difference equations given here approximations are made to the operator, $\nabla \times \nabla \times$, acting on the vector, $\overrightarrow{\mathrm{H}}_{\theta}$, and to $\nabla^{2}$ acting on the scalar, $T$. 


\section{Method}

The fundanental equatione at: organiced so that changes of variables associated with a mass puint cun be interpreted as due to a flux through a surfuce surroulding the mass point. Thi difference tperators are orgyized in the same way. j.c... in tle spiril of the Jivergence theorem. Space derivatives in two dimensions are defined as the summation of the normal components of the Mux around an enclused area. Thus. fiur a vector. $F$, repre senting a flux (e.g., magnetic, Jeat flows, velosity, etc.). the following tr.tegral defintions of the partial derivatives are useci. See Fig. 1.

$$
\begin{aligned}
& \frac{\partial F}{\partial \bar{X}}=\lim _{A \rightarrow C} \int_{(C)} \frac{F(\vec{n} \cdot \vec{j}) d l}{A} \\
& \frac{\partial F}{\partial Y}=\lim _{A \rightarrow n} \int_{(C)} \frac{F(\vec{n} \cdot \vec{j}) d Q}{A}
\end{aligned}
$$

where

$$
\begin{aligned}
& d Q=\text { arc lengtl } \\
& (C)=\text { boundary of area } A \\
& \vec{n}=\text { normal vector } \\
& \vec{T}=\text { tangent vector }
\end{aligned}
$$

The material is divided into a Lagrange grid that moves with the flow. The intersection of the grid lives will be called zone node puints, and the interior space of the mesh called zones. The integration path for evaluaning the partial derivatives is defiued i: the grid in two ways. Figure 2 shows the integration path for evaluar ting compunents of the vector, $\nabla \times \overrightarrow{\mathbf{H}}$. Referring to Fig. 2, if $\mathrm{F}$ is a lunction (in this case, $\mathrm{F}=\mathrm{H}_{\theta}$ ) licfined at zone centers, then the difference eyuations for evaluating $\frac{\partial F}{\partial X}$ and $\frac{\partial F}{\partial Y^{\prime}}$ centered at point $I$ are:

$$
\left.\frac{\partial F}{J X}\right|_{Q}=\frac{\int(C) F(\vec{r} \cdot \vec{i}) d r}{\bar{A}}
$$

where

$$
\begin{aligned}
& \left.\int_{(C)} F_{I} \vec{\cdots} \cdot \vec{i}\right) l l=-\left[F_{W}\left(Y_{I I}-Y_{I I I}\right)+F_{(2)}\left(Y_{I I I}-Y_{I V}\right)\right. \\
& \left.+F_{(3)}\left(Y_{I V}-Y_{1}\right)+F_{(4)}\left(Y_{I}-Y_{1]}\right)\right] \text {, }
\end{aligned}
$$

and

$$
\left.\frac{\partial F}{\partial Y}\right|_{O D}=-\frac{\int_{(C)} \Gamma(\vec{n} \cdot \vec{j}) d x}{A}
$$

where

$$
\begin{aligned}
& \int_{(C)} F(\vec{n} \cdot \vec{j}) d l=+\mid F_{\Phi}\left(X_{I 1}-X_{I I 1}\right)+F_{(\Phi)}\left(x_{I I I}-X_{1 V}\right) \\
& +F_{(3)}\left(x_{1 V}-x_{1}\right)+F_{(4)}\left(x_{1}-x_{I I}\right) i
\end{aligned}
$$

$A$ is the area enclused by the integration path, I, II. III, IV (Fig. 2). and is taken as the average of the area cuntrihutions of the four zones surrusuding point 1 (Fig. 2).

Figure 3 shows the patl for evaluating $\nabla \times \nabla \overrightarrow{\mathbf{H}}$. If $F$ is a function (in this wse a component of the vector $\nabla \times \vec{H})$ defined at zone nodes, then the difficrence equations for evaluating $\frac{\partial F}{\partial X}$ and $\frac{\partial F}{\partial Y}$. defined at a zons s.nler, are:

$$
\begin{aligned}
\left.\frac{\partial F}{\partial \mathrm{X}}\right|_{D}= & \frac{\int_{(i)} F(\vec{n} \cdot \vec{i}) d Q}{A} \\
= & -\frac{I}{A}\left\{F_{23}\left(Y_{2}-Y_{3}\right)+F_{34}\left(Y_{3}-Y_{4}\right)\right. \\
& +F_{41}\left(Y_{4}-Y_{1}\right)+F_{12}\left(Y_{1}-Y_{2}\right) ! \\
= & \left.+\frac{1}{2 A} i\left(F_{2}-F_{4}\right)\left(Y_{3}-Y_{1}\right)-\left(i_{2}-Y_{4}\right)\left(F_{3}-F_{1}\right)\right\} .
\end{aligned}
$$

Similarly,

$$
\left.\frac{\partial \mathrm{F}}{\partial Y}\right|_{\mid \omega}=-\frac{1}{2 A}\left[\left(F_{2}-F_{4}\right)\left(X_{3}-X_{1}\right)-\left(X_{2}-X_{4}\right)\left(F_{3}-F_{1}\right)\right]
$$

Here, $F_{23}=\left(F_{2}+F_{3}\right) / 2$, etc., and $A$ is the area of zone $\Phi$ defined by points $1,2,3,4$. 


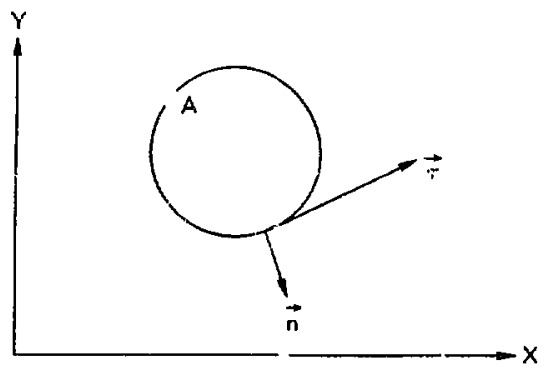

Fig. I. Integration scheme.

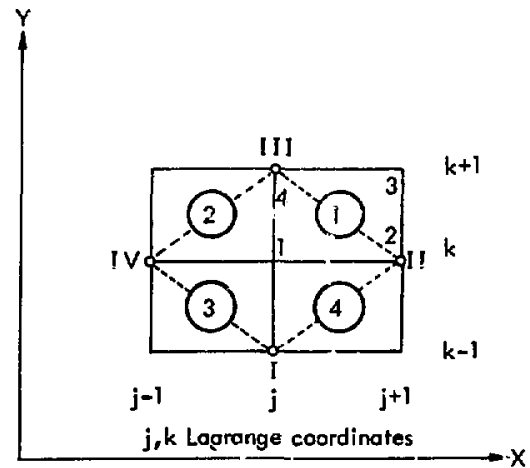

Fig. 2. Grid for calculating $\nabla \times \vec{H}$, where $\overrightarrow{\mathrm{B}}$ is given in a zone centes. Dotted line shows integration path for

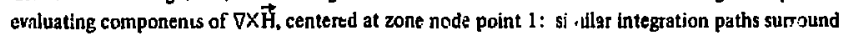
polnts 2,3 , and 4 . 


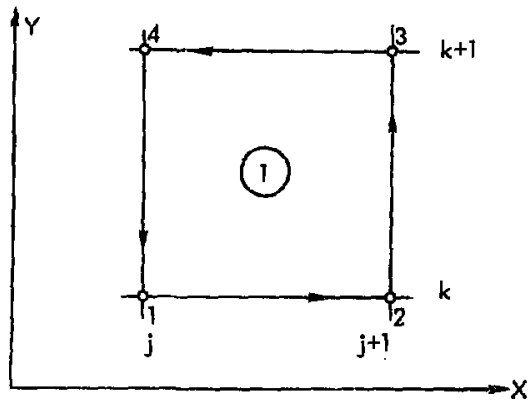

Fig. 3. Grid for calculating $\nabla \times \nabla \times \overrightarrow{\mathrm{H}}$, where $\nabla \times \overrightarrow{\mathrm{H}}$ is given at node points. Arrows show integration path where $\nabla \times \nabla \times \vec{H}$ is centered at $j+l / 2, k+1 / 2$ (zone center).

\section{Fundamental Equations of Magnetohydrodynamics}

The following equations are solved in Lagrenge cuor dinates with 2-D cylindricul symmetry, using the structure of the HEMP code:

\section{LQUATION OF MOTION}

$$
\rho \frac{\mathrm{d} \overrightarrow{\mathrm{W}}}{\mathrm{dt}}=\nabla \overrightarrow{\mathrm{L}}+\left\{\mu_{\mathrm{In}} \overrightarrow{\mathrm{J}} \times \overrightarrow{\mathrm{H}}\right\}
$$

As shown in the following section, the term in braces [Lirentz force] is incorporated into the lotal stress tensor, $\overrightarrow{\mathbf{s}}$, of the HEMP proygum.

\section{ELICTROMAGNETIC FIELD EQUATJONS}

Faraday's Law

$$
\mu_{\mathrm{m}} \frac{\mathrm{d}}{\mathrm{dt}} \iint_{\mathrm{S}} \overrightarrow{\mathrm{H}} \cdot \overrightarrow{\mathrm{n}} \mathrm{ds}=-\int_{(C)} \overrightarrow{\mathrm{E}} \cdot \overrightarrow{\mathrm{d} R}
$$

Ampere's Law

$$
\nabla \times \vec{H}=4 \pi \overrightarrow{\mathbf{J}}
$$

$$
\frac{\text { Ohun's Law }}{\vec{E}=\frac{\vec{J}}{\vec{C}}}
$$

By Stoke's theorem and substitution of Eqqs. (3) and (4). Eq. 2 can be written as:

$$
\begin{aligned}
& \mu_{\mathrm{m}} \frac{\mathrm{d}}{\mathrm{dt}} \iint_{S} \overrightarrow{\mathrm{H}} \cdot \vec{\pi} \mathrm{ds}=\int_{(C)} \overrightarrow{\mathrm{E}} \cdot \overrightarrow{\mathrm{d} l} \mathrm{l}=-\iint_{S} \nabla \times \overrightarrow{\mathrm{E}} \cdot \overrightarrow{\mathrm{n}} \mathrm{ds} \\
& \quad=-\iint_{S} \nabla \times\left(\frac{1}{4 \pi \mathrm{C}} \nabla \times \overrightarrow{\mathrm{H}}\right) \vec{n} \mathrm{ds}
\end{aligned}
$$

The above rebations apply to a local region of the fluid that moves with the fluid. Applying Eq. (5) to the Lagrange region and considering an $\mathbf{H}_{\theta}$ field only gives the inganetic flux diffusion equation:

$$
\mu_{\mathrm{m}} \frac{\mathrm{d} \Phi}{\mathrm{dt}}=-\nabla \times\left(\frac{1}{4 \pi \mathrm{C}} \nabla \times \mathrm{H}_{\theta}\right) \mathrm{A}
$$

$$
\mu_{m} \Phi=\mu_{m} H_{\theta} A=\text { magnetic flux }
$$

$\mathrm{H}_{\theta}$ = theta component of magnetic field, $\overrightarrow{\mathrm{H}}$

A = surface element, defined later as the area. is Lagrange zone.

It is in the form of Eq. (6) that Faraday's law, Ampere's law, and Ohm's law are used in the calculstion. It is noted that the displacement cunent has 
been unitied and that $\nabla \cdot \mu_{m} \vec{H}=0$ is identically satisfied for the cise considered here with $\overrightarrow{\mathbf{H}}=\mathrm{H}_{0}$ unly and symmetry in the $\theta$ direction.

\section{ENERGY EQUATION}

The total changs in interva! energy, dit, in a qiven volume is:

$$
\begin{aligned}
d E= & -(P+q) d V+V\left\{\nabla \cdot\left(\left(K+\frac{4}{3} \cdot R^{c} \lambda T^{3}\right) \nabla T\right)\right\} d t \\
& +V \frac{J^{2}}{C} d t+d z
\end{aligned}
$$

where:

$$
\begin{aligned}
\mathrm{d} Z & =\mathrm{VT} \mathrm{xy}_{x y} \dot{\epsilon}_{x y} \mathrm{dt}+\mathrm{v}\left(\mathrm{s}_{x x}-\frac{\mu_{m}}{8 \pi} \mathrm{H}^{2}\right) \dot{\epsilon}_{x x} d t \\
& +\mathrm{v}\left(c_{y y} \frac{\mu_{m}}{8 \pi} H^{2}\right) \dot{\epsilon}_{y y} d t+v\left(s_{\theta \theta}+\frac{\mu_{m}}{8 \pi} H^{2}\right) \dot{\epsilon}_{\theta \theta} d t .
\end{aligned}
$$

The first term on the right in Lq. (7) is the change in Inierrial energy from pressure forces; the second term is the energy change from thermal conduction and radiation flow: the third term is the energy change from ohmic heating; and the fourth term, from distortion stresses.

The following definitions are used: $E=c_{v} T+a_{R} \sqrt{T^{4}}=$ material energy + radiation energy

$$
d E=\left.\frac{\partial E}{\partial T}\right|_{V} d T+\left.\frac{\partial E}{\partial V}\right|_{T} d V
$$

(i) $\left.\frac{\partial E}{\partial T}\right|_{V}=\left.\frac{\partial^{m} E}{\partial T}\right|_{V}+\operatorname{ta}_{R} V T^{3}$

(ii) $\left.\frac{\partial E}{\partial V}\right|_{T}=\left.\frac{\partial^{m_{E}}}{\partial V}\right|_{T}+a_{R} T^{*}$

Here, $m_{\epsilon}=$ material energy $=c_{v} T$.

Substitution of Eq. (9) into Eq. (8) gives:

$$
\begin{aligned}
\left.d T \frac{\partial E}{\partial T}\right\}_{V}= & -\left(P+\left.\frac{\partial E}{\partial V}\right|_{T}+q\right) d V+(d t V) P=(A \\
& +\frac{V)^{2}}{C} d t+d Z
\end{aligned}
$$

where:

$$
\begin{aligned}
\hat{A} & =K+\frac{4}{3} \mathrm{a} \mathrm{R}^{c \lambda T^{3}} \\
\mathrm{dZ} & =\text { cliange in internal energy from distortion stresses. }
\end{aligned}
$$

Rewriting Eq. (10):

$$
\left.\mathrm{dT} \frac{\partial \mathrm{E}}{\partial \mathrm{T}}\right|_{\mathrm{V}}=\mathrm{V}[\nabla \cdot(\Lambda \nabla \mathrm{T})] \mathrm{dt}+\mathrm{W}
$$

where:

$$
\begin{aligned}
& W=-\left(P+\left.\frac{\partial E}{\partial V}\right|_{T}+q\right) d V+\frac{V J^{2}}{C} d t+d Z \\
& P=\text { material pressure }+\frac{l}{3} a_{R} T^{4} .
\end{aligned}
$$

It is in the form of Eq. (11) that changes in the internal energy are calculated. Equation (1 1 ) is a diffusion equation for the temperature. $T$.

\section{CONTINUITY EQUATION}

$$
\nabla \cdot \vec{W}=\frac{\dot{V}}{V}
$$

\section{CONSTITUTIVE RELATIONS}

$$
\begin{aligned}
& P \quad=P_{m}+P_{R} \quad=\text { total pressure } \\
& P_{m}=P_{m}(T, V) \quad=\text { material pressure } \\
& P_{R}=\frac{l}{3}{ }^{a} R^{T^{4}} \quad=\text { radiation pressure } \\
& \mathrm{K} \quad \mathrm{K}(\mathrm{T}, \mathrm{V}, \mathrm{H}) \quad=\text { thermal conductivity } \\
& \lambda \approx \lambda(T, V) \quad=\text { Rosseland mean free path } \\
& c_{v}=c_{v}(T, V) \quad=\text { specific heat } \\
& c=C(T, V) \quad=\text { electrical conductivity } \\
& m_{E}=c_{v} T \quad=\text { material internal energy }
\end{aligned}
$$

$\left.\frac{\partial E}{\partial T}\right|_{V}=\left.\frac{\partial m_{\epsilon}}{\partial T}\right|_{V}+4 a_{R} T^{3}=\begin{aligned} & \text { change in intemal energy } \\ & \text { with respect to temperature }\end{aligned}$

$\left.\frac{\partial E}{\partial V}\right|_{T}=\left.\frac{\partial^{m_{E}}}{\partial V}\right|_{T}+a_{R} T^{4}=\begin{aligned} & \text { change in intemal energy } \\ & \text { with respect to volume }\end{aligned}$

The above relationships must be given in advance in the form of explicit? ?unctions or table look-ups. 


\section{Difference Equations for Magnetohydrodynamics}

EQUATIONS OF MOTION

$$
\rho \frac{d \vec{W}}{d t}=\nabla \vec{\xi}+\mu_{m} \vec{J} \times \vec{H}
$$

Lorentz lusce (stfect of the mannetic ficld on the Aluit motion):

$$
\mu_{m} \vec{J} \times \overrightarrow{: t}=\frac{\mu_{m}}{4_{n}}(\nabla \times \vec{H}) \times \vec{H} .
$$

Using $X \cdot Y$ soordinates with cylindrical symmetry ahout the $X$-ixis, and considering a fietd. $H$, in the theta direction $\mathrm{H}_{0}$ only, Fq. (12) hoconses:

(a)

$$
\left\{\begin{array}{l}
\rho \ddot{\mathrm{X}}=\frac{\partial \Sigma_{\mathrm{xx}}}{\partial \overline{\mathrm{X}}}+\frac{\partial \mathrm{T}_{\mathrm{xy}}}{\partial \overline{\mathrm{Y}}}+\frac{\mathrm{T}_{\mathrm{xy}}}{\mathrm{Y}}+\left(-\frac{\left.\mu_{\mathrm{m}} \frac{\partial \mathrm{H}^{2}}{\partial \mathrm{X}}\right)}{\partial \mathrm{X}}\right) \\
\rho \ddot{\mathrm{Y}}=\frac{\partial \mathrm{T}_{\mathrm{xy}}}{\partial \mathrm{X}}+\frac{\partial \Sigma_{y y}}{\partial \bar{Y}}+\frac{\Sigma_{\mathrm{yy}}-\Sigma_{\theta H}}{\mathrm{Y}}+\left(-\frac{\mu_{\mathrm{m}}}{8 \pi} \frac{\partial H^{2}}{\partial \mathrm{Y}}-\frac{\mu_{\mathrm{m}}}{4 \pi} \frac{\mathrm{H}^{2}}{\mathrm{Y}}\right)
\end{array}\right.
$$

or:

(b)

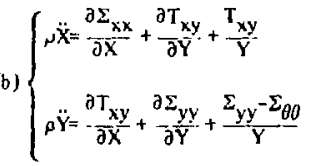

where:

$$
\begin{aligned}
& s_{x x}=-P+s_{x x}-\frac{\mu_{m}}{8 \pi} H^{2} \\
& s_{y y}=-P+s_{y y}-\frac{\mu_{m}}{8 \pi} H^{2} \\
& \Sigma_{\theta \theta}=-P+s_{\theta \theta}+\frac{\mu_{m}}{8 \pi} H^{2}
\end{aligned}
$$

It is seen llat the magnetic force is like a pressure in the $X$ and $Y$ directions, and like a tension in the thets direction. With the addition of tlie ahove pressure. like terns from the magnetic field. there are no uther clanges to the finite difference cquations of motion of the HEMP code.

\section{MAGNETIC DIFFUSION}

Calculation of $d d / \mathrm{d} t$ centered in space it $\mathrm{j}+1 / 2$, $k+1 / 2,\left(\right.$ see Fig. 4) and in time at $t^{n+1}$ :

$$
\mu_{\mathrm{III}} \underset{\mathrm{di}}{\mathrm{di}}=-\nabla \times\left(\frac{1}{4 \pi \mathrm{C}} \nabla \times \vec{H}\right) A
$$

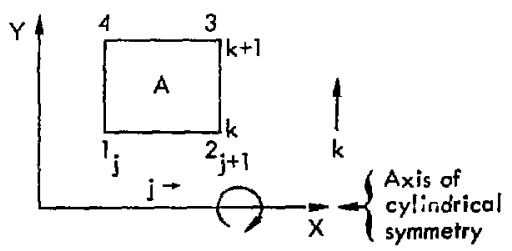

Fig. 4. Lagrange zone defined by points $1,2,3$, and 4 .

$$
\begin{aligned}
& \overrightarrow{\mathrm{H}}=\mathrm{H}_{\theta} \text { unly } \\
& \mathbf{A}=\text { zone area } \\
& \mathrm{C}=\text { conductivity } \\
& \mathbf{W}=\mathbf{H A} \\
& \mu_{\mathrm{In}}=\text { magnetic permeability }
\end{aligned}
$$

\section{Procedure}

a. The vector $\nabla \times \vec{H}$ is evill ated at zone node points $1,2,3$ and 4 , using the difference stheme of the preceding tection, where $\vec{H}$ is considered defined at a zone center (see Fig. 4).

b. The requiru'd value of $\nabla \times\left(\frac{1}{4 \pi \mathrm{C}} \nabla \times \vec{H}\right)$ defined at $\mathrm{j}+1 / 2, k+1 / 2$ (zone center) is obtained by averaging the components of the vector $\nabla \times \vec{H}$. so that they are defined at the middle of the line connecting consecutive $j . k$ points and integrating around the area $A$, as discussed in the preceding section. A representative value of the conductivity, C, for each leg of the integration path is obtained by an averaging scheme based on curremt flow in parallel circuits.

c. A forward difference schene is used which motan: that the desired value of $\mathrm{H}^{\mathrm{n}+1}$ at time. $\mathrm{t}^{\mathrm{n+1}}$, is an implicit function of $\mathbf{H}^{\mathrm{n}+1}$, i.e..

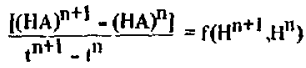


Fut a given $j$ litic. the value of $H_{j+1 / 2, k+1 / 2}^{\prime \prime+1}$ sian be fomd by solving as set of linedr equations if we firs! evaluate $\nabla \times \vec{H}$ at points 1.2 .3 and 4 , using values of $\mathrm{H}^{11+1}$ for all k-liues civirespunding (1) the given $\mathrm{j}$-line

(Fig. 5). The change of nux. $\mu_{m}$ d $\Phi$ is then dsterItinted by using unly sides $1-2$ and $3-4$ in step ( 17 ) ahove. The conductivity, $C$, associaled $u$ ith a side is oblained from the conductivity of the zones on either side of the line being considesed. The calculated value of $\mu_{m} \mathrm{dc}$ will he the cllange is flux in the $k$-direction. Details of the calculation are given in Appendix l.s.

d. The vilculation of $\nabla \times \vec{H}$ is repeated for points $1,2,3$ and 4, but this time using values of $\mathrm{H}^{\mathrm{n+1}}$ for all j-lines corresponding to a given $\mathrm{k}$-line (Fig. 6). The change ill nux. $\mu_{\mathrm{II}}$ dw is determined as in step (c), but this time using unly sides $2 \cdot 3$ and $4-1$. This will be the change in fux in the j-direction. See Appendix l,b.

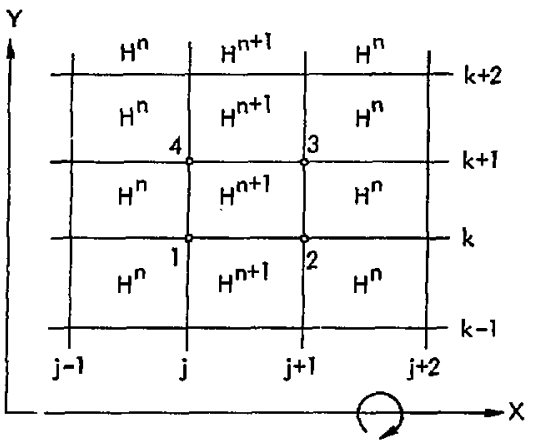

Fig. 5. Grid showing the time centering of $\overrightarrow{\mathbf{H}}$ used to evaluate the components of $\nabla \times \overrightarrow{\mathbf{H}}$ for calculating the flux change in the $\mathrm{k}$-direction, where $\mathrm{j}$ and $\mathrm{k}$ are Lagange coordinates.

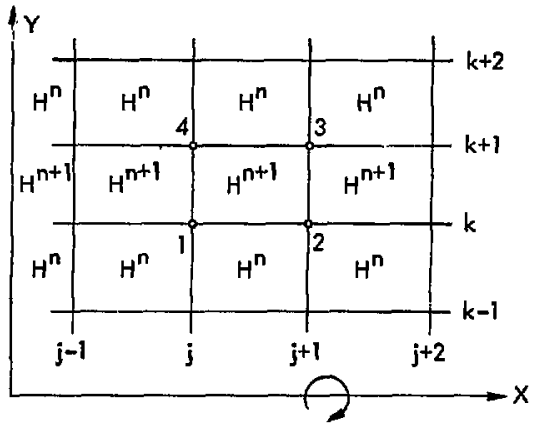

Fig. 6. Grid showing time certering of $\vec{H}$ used to evaluate the components of $\nabla \times \vec{H}$ for calculating the flux change in the $\mathrm{j}$-direction. 
e. The total chamge int fux, $\mu_{\mathrm{n}}$ 't, for the zone $j=1 / 2, k+1 / 2$ is oblained by udding togetlier the flux change in the two directions: i.e., fotal fux change. $\mu_{m} d \Phi=\mu_{m}(d, J)^{k}+\mu_{1 n}(d \Phi)^{j}$, where $(\mathrm{d} \Psi)^{k}$ is obtaincd fron step (c), and $(d w){ }^{j}$ is obtained from step (d).

r. Tle desired value of $H^{n+1}$ is obtained from step (e):

$$
\begin{aligned}
& (H A)^{n+1}-(H A)^{n}=\left[(H A)^{n+1}-(H A)^{n}\right]^{k} \\
& +\left[(H A)^{n+1}-(H A)^{n}\right]^{j} \\
& {\left[(H A)^{n}\right]^{k}=\left[(H A)^{n !}\right]^{j}=(H A)^{n}} \\
& {\left[I^{n+1}=\left(H^{n+1}\right)^{k}+\left(H^{n+1}\right\}^{j}-\left(\frac{H^{n} A^{n}}{A^{n+i}}\right)\right.}
\end{aligned}
$$

When specified to one spuce dimension, the space and time-centering of the difference equations corsespond to the Crank.Nichulsun differencing sclesmc. ${ }^{2}$

\section{ENERGY EQUATIONS}

Temperature [iffusion

Rewriting La. (11) with the quantities at times n and $n+1 / 2$ considered to be kitown. where the un. known quantity is $T^{11+1}$, gives:

$$
\underset{+W^{\prime l}}{\left(T^{11+1}-T^{n}\right)\left(\frac{\partial E}{\partial T} \mid v\right)^{n}=\Delta t^{n+\frac{1}{2}} v^{n+1}\left(\nabla \cdot \wedge^{n} \nabla T\right)}
$$

where $\nabla T$ is calculdled with $T^{n}$ jad $T^{n+1}$. The temperature. $T$, is centered at $j+1 / 2, k+1 / 2$ (zone (enter).

a. Equation $(16)$ is solved in the same miunnet as the magnetic diffusion equet tivil. Eq. (15), 10 obain new temperatures $\left(T^{n+1}\right)^{k}$ and $\left(T^{n+1}\right)^{j}$ from the tempera ture diffusion in the $k$ and $j$ directions. respectively. Details of these colculations are given i1 Appendix B.

b. The total entergy charge is the sunt of contributions from the $k$ plus the direstions. The value of $\partial \mathrm{E} / \mathrm{aT}$ is considered constant during the tim: interval. $t^{n} \rightarrow t^{11+1}$. The new temperature for a zone is obtained is follows:

$$
\begin{aligned}
& T^{n+1}-T^{n}=\left(T^{n+1}\right)^{k}-T^{n}+\left(T^{n+1}\right)^{j}-T^{n} \\
& \therefore T^{n+1}=\left(T^{n+1}\right)^{k}+\left(T^{n+1}\right)^{j}-T^{n} .
\end{aligned}
$$

This temperature is considered to be the first approximation to the desirel temperature and will be designated as $\tilde{\mathbf{T}}^{1 \mathrm{t}+1}$.

c. The pressure, $\tilde{\mathrm{p}}^{\mathrm{n}+1}$, and $(\partial \tilde{\mathrm{E}} / \partial V)^{n+1}$ are calculated from the given constitutive relations using $\tilde{T}^{n+1}$ and $v^{n+1}$. The quantity, $W_{1}$ is then recalculated using $\mathrm{P}^{\mathrm{N}+1 / 2}$ and $(\partial \mathrm{E} / \partial \mathrm{V})^{\mathrm{n}+1 / 2}$, with the other quantities remaining the same; where,

$$
\begin{aligned}
& \mathrm{p}^{n+1 / 2}=1 / 2\left(\mathrm{P}^{n+1}+\mathrm{P}^{n}\right), \\
& (\partial \mathrm{E} / \partial V)^{n+1 / 2}=1 / 2\left[(\partial \mathrm{E} / \partial V)^{n+1}+(\partial E / \partial V)^{n} \cdot\right]
\end{aligned}
$$

d. The quantity (DE/aT) $)^{n+1 / 2}$ is calculated from the given constitutive relation. The tine-centering of $T$ is given by:

(i) $T^{n+1 / 2}=1 / 2\left(\tilde{T}^{n+1}+T^{n}\right)$

(ii) $\left(T^{n+1 / 2}\right)^{3}=\frac{1}{4}\left[\left(\tilde{T}^{n+1}\right)^{3}+\left(\tilde{T}^{n+1}\right)^{2} \cdot\left(T^{n}\right)\right.$

$$
\left.+\left(\mathrm{T}^{\mathrm{n}+1}\right)\left(\mathrm{T}^{\mathrm{k}}\right)^{2}+\left(\mathrm{T}^{\mathrm{a}}\right)^{3}\right\} \text {. }
$$

This definition of $\mathrm{T}^{3}$ assures that

$$
d E \approx\left(c_{y}+4{ }_{d} V T^{3}\right) d T \text {, }
$$

if $c_{v}$ and $V$ are constant in ths tinje interval between $n$ and $n+!$.

c. Steps (a) and (b) are repeated using up-lated values of $\partial E / \partial T$. $A$. and $W$ obtaincd from the tine. ceatering given, ? steps (c) and (d), and the constitur tive relations.

\section{Space-Centering of Parameters at the Interfaces of Conseculine Znnes.}

In Ilic integatiun scheme, representalive values of parametess caleulated fram the constitutive relations 
are required for eac h leg of the integration patls. The averaging technique used is given below.

Consider the leg 1.2 of the integration path 1,2,3, 4, 1 around zone (5) in Fig. 7.

Coefficient of thermal conduction, $\mathrm{K}$ -

$$
K_{1-2}=\frac{A_{(5}+A_{(2)}}{\frac{A_{(5)}}{K_{(5)}}+\frac{A_{(2)}}{K_{(2)}}} \quad A=\text { zone area }
$$

Rosseland mean rree path, $\lambda$ -

$$
\lambda_{1-2}=\frac{A_{0}+A_{Q}}{\frac{A_{(9)}}{\lambda_{(5)}}+\frac{A_{(9)}}{\lambda_{(9)}}}
$$

To insure the thermal flow is from high to low temperature zoncs, $\lambda_{B} *$ and $\lambda_{D} *$ are calculated using the maximum temperature of the zones (2) and (5) (Ref. 3).

Transmissivity, $\Lambda$ -

$$
\Lambda_{1-2}=K_{1-2}+\left(\frac{4}{3} a^{c}\right) \cdot \lambda_{1-2} \cdot T_{1-2}^{3}
$$

where

$$
\mathrm{T}_{\mathrm{1} \cdot 2}^{3}=\frac{1}{4}\left(\mathrm{~T}_{\Theta}^{2}+\mathrm{T}_{\theta}^{2}\right)\left(\mathrm{T}_{\theta}+\mathrm{T}_{\theta}\right)
$$

Electrical conductivity $\mathrm{C}$ -

$$
c_{1.2}=\frac{\left(\mathrm{CV} \frac{M}{\rho_{0}}\right)_{\odot}+\left(\mathrm{CV} \frac{M}{\rho_{0}}\right)_{\varrho}}{\left(v \frac{M}{\rho_{0}}\right)_{\odot}+\left(v \frac{M}{\rho_{0}}\right)_{\circledast}}
$$

where $M=$ zone mass.

Similar expressions are written for legs $2-3,3-4$ and $4-1$.

Olınic Heating, $\frac{\mathrm{J}^{2}}{\mathrm{C}}$

$$
\begin{aligned}
\frac{j^{2}}{\mathrm{C}} & =\frac{1}{16 \pi^{2} \mathrm{C}}(\nabla \times \vec{H})^{2} \\
& =\frac{1}{16 \pi^{2} \mathrm{C}}\left(a^{2}+b^{2}\right)
\end{aligned}
$$

where $\nabla \times \vec{H}=\vec{a} \vec{i}+\vec{b} \vec{j}$.

Referring to Fig. 7, the ohmic heating for zone (4) is given by:

$$
\left(\frac{\mathrm{s}^{2}}{\mathrm{c}}\right)_{(3)}=\left[\frac{1}{2}\left(\frac{\mathrm{s}^{2}}{\mathrm{c}}\right)_{0}^{\mathrm{k}}+\left(\frac{\mathrm{J}^{2}}{\mathrm{c}}\right)_{0}^{\mathrm{j}}\right]
$$

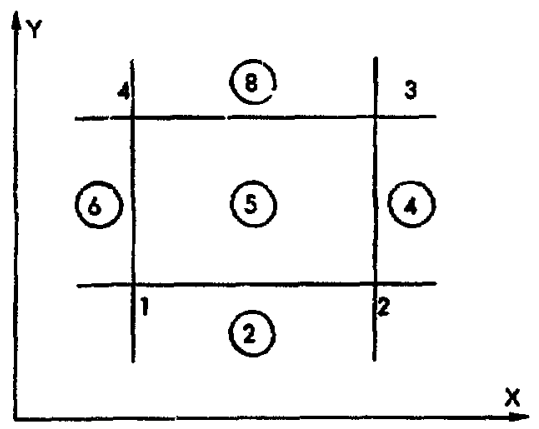

Fig. 7. Nodes $1,3,3$, and 4 and zoncs $0,0,0$, und 9 surrounding zone 0 . 
where:

$$
\begin{aligned}
& \left(\frac{b^{2}}{c}\right)_{(3)}^{k}=\frac{c_{B}}{2 \cdot 16 \pi^{2}}\left[\left(\frac{a_{1}^{2}+a_{2}^{2}}{2}+\frac{b_{1}^{2}+b_{2}^{2}}{2}\right)\left(\frac{1}{c_{1-2}}\right)^{2}\right. \\
& \left.+\left(\frac{a_{3}^{2}+a_{4}^{2}}{2}+\frac{b_{3}^{2}+b_{4}^{2}}{2}\right)\left(\frac{1}{c_{3-4}}\right)^{2}\right] \\
& \left(\frac{\mathrm{J}^{2}}{\mathrm{C}}\right)_{\mathfrak{G}}^{\mathrm{j}}=\frac{\mathrm{C}_{\mathfrak{G}}}{2 \cdot 16 \pi^{2}}\left[\left(\frac{\mathrm{a}_{2}^{2}+\mathrm{a}_{3}^{2}}{2}+\frac{\mathrm{b}_{2}^{2}+\mathrm{b}_{3}^{2}}{2}\right)\left(\frac{1}{\mathrm{C}_{2-3}}\right)^{2}\right. \\
& \left.+\left(\frac{a_{4}^{2}+a_{1}^{2}}{2}+\frac{b_{4}^{2}+b_{1}^{2}}{2}\right)\left(\frac{1}{C_{4-1}}\right)^{2}\right]
\end{aligned}
$$

The superscripts $k$ and $j$ refer to the $k$ and $j$ sweeps described earlier.

\section{CONTINUITY EQUATION}

Mass is conserved explicitly in the Lagrange formulation of the fundamental equations.

The continuity equation

$$
\nabla \cdot \vec{W}=\frac{\partial \dot{X}}{\partial \dot{X}}+\frac{\partial \dot{Y}}{\partial \bar{Y}}+\frac{\dot{Y}}{Y}=\frac{\dot{V}}{V}
$$

is used to desuribe components vi the strain tensor and is explained in Ref. 1.

\section{T.ME.STEP CONTROL}

The time step is taken as the minimum over the grid mesh of the mesh size divided by the largest wave speed (Courrant condition). This choice of time-step can insure stability of the difference equations, but does not insure accuracy of the diffusion calculations. In the diffusion calculations a much smaller time-step is required in order to provide communication of the boundary conditions throughout the grid.

An extrapolation method devised by Julius Chang and Jim LeBlanc permits a large time-step to be used, but still maintains the accuracy of a much smaller time-step. The application specinlized to one dimension is discussed in Ref. 4. This sclieme provides a considerable saving in computation time in the wolution of diffusion equations by the double sweep techinique described hare.
With an equation of the form

$$
\frac{F^{n+1}-F^{n}}{\Delta t}=f\left(F^{n+1}, F^{n}\right)
$$

this extrapolation method advances in time the parameter $F$ from $F^{n}$ to $F^{n+1}$. Using the double sweep technique, solve the implicit equation three times, as follows:

$$
\begin{aligned}
& \text { (a) } \frac{F^{i}-F^{n}}{\Delta t}=f\left(F^{i}, F^{n}\right) \\
& \text { (b) } \frac{F^{i j}-F^{n}}{\frac{\Delta t}{2}}=f\left(F^{i i}, F^{n}\right)
\end{aligned}
$$

(c) $\frac{\mathrm{F}^{\mathrm{iii}}-\mathrm{F}^{\mathrm{ii}}}{\frac{\Delta t}{2}}=f\left(\mathrm{E}^{\mathrm{iii}}, \mathrm{F}^{\mathrm{ii}}\right)$

Then $F^{n+1}=2 F^{i i i}-F^{i}$.

\section{BOUNDARY CONDITIONS}

The magnetic field, temperature and pressure 112ust be specified for all times on the exterior boundaries of the material region being considered. Usually the pressure is assumed zero on exterior surfaces. For problems where the material occupies a region containing the axis of symmetry, the boundary conditions for the axis of symmetry are:

$$
\begin{aligned}
& \mathrm{H}_{\theta}=0 \\
& \frac{\partial \mathrm{P}}{\partial \mathrm{Y}}=\frac{\partial \mathrm{T}}{\partial \mathrm{Y}}=0,
\end{aligned}
$$

where $\mathrm{Y}$ is the radial coordinste. For symmetry planes perpendicular to the $\mathrm{X}$-axis:

$$
\frac{\partial \mathrm{H}_{\theta}}{\partial \mathrm{X}}=\frac{\partial \mathrm{P}}{\partial \mathrm{X}}=\frac{\partial \mathrm{T}}{\partial \mathrm{X}}=0 .
$$

For the application of boundary conditions, it is convenient to provide phantom zones around the region of interest. The zero gradient conditions above are achieved by reflecting the values of the interior parameters into the phantom zones. 


\section{SLIDING INTERFACES}

For most problems of practical interest, it is important to allow for one material to slide upon another. The details of the computer logic for sliding interfaces in the framework of elastic-plastic flow are given in Ref. 1 . In the solutions of the diffusion equations presented here, it is seen that the zones nust be continuous in the Lagrange zone directions, $j$ and $k$. This is accomplished by subdividing the grid just before the diffusion calculations are made.

Figure 8 shows a sliding in terface that separates regions $A$ and $B$. The slide line logic of the HEMP program that is required to develop in the computer the geometry corresponding to Fig. 8 is described in Ref. 1. As shown by the dotted lines in Fig. 8 the grid of region $B$ is extended through region $A$. Thus, tegion $\mathrm{A}$ is now considered to be mapped by the original $k$ lines of region $A$ and the $j$ lines of region $B$. The new $j$ grid of region $A$ is generated so that it intersects the $\mathrm{k}$ lines in the same proportions to the original $j$ lines as occurred on the slide line. The new grid will overlay one or more zones of the old grid of region A. A single value of the parameters $H$ or $T$ is obtained for each zone of the new grid by an area weighting sclieme. For example if a zone of the new grid is composed of parts of zone $a$ and zone $b$, see Fig. 8, then the appropriate value of the parameter $H$ for the new grid is

$$
H=-\frac{H_{a} A_{a}+H_{b} A_{b}}{A_{a}+A_{b}} \quad \text { (similar for } T \text { ) }
$$

Here $A_{a}$ and $A_{b}$ refer to the area of the new grid that overlay zones $\mathrm{a}$ and $\mathrm{b}$ respectively of ti.e old grid.

Thus, the furm of the original calculational scheme is established and the same method can be used to solve the implicit equation for new values of the magnetic field $\mathrm{H}$ and the temperature T. After the diffusion calculations are completed, the updated parameters are reassigned to the solid line grid by an area weighting scheme similar to the above.

In this example the zones of region $B$ have been used to define zones in region $A$. The same loglc could have been applied using region $A$ to define zones in region $B$.

It is seen in Fig. 8 that region $A$ extends beyond region $B$. The problem requires that the boundary conditions are alwoys known in advance of a time step calculation. This informetion is carried in the

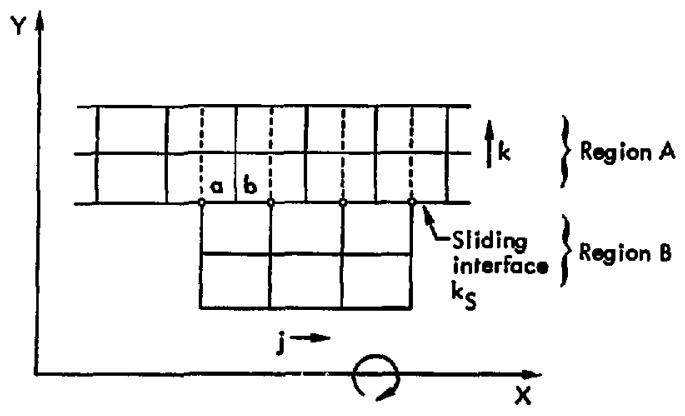

Fis. 8. Gid for calculating ficld and temperature diffusion across a aljing interface.

(a) Solid lines - Lagrange grid at end of equation of motion calculation;

(b) An ordered grid is created by the dotted lines for the diffusion cilculations. 
computer logic by pseudo zones that surround the entire calculational grid. The use of pseudo zones permits parameters on the grid boundaries, including those of the overhang of region A, Fig. 8 to be calculated in the same manner as any other point in the grid. (A slide line extension is required if points associated with region $B$ on the slide line $k_{s}$ move beyond the ends of tegion $A$.)

The example shown nere has considered a single sliding surface along a $k$ line. The same appraoch is used if more than one $k$ line is a slide line. The region that is to control the zoning for the diffusion calculations nust be stated in advance.

Figure 9 shows a hydrodyngmic ca'culation set up with slide lines in two directions ( $j$ and $k$ slide lines).
This provision in the computer program is very useful for permitting a fine zoned region to be defined in the two dimensional grid. The same mapping scheme for calculating the temperature and $v$ : magnetic diffusion is used, the only difference being that additional imapping is required so as to include all material regions surrounding a given region, i.e., both the k-lines and the $\mathrm{j}$-lines are extended from the region that controls the zoning.

\section{CHECK PROBLEMS}

The accuracy of the numerical technique that calculates the diflusion of $T^{4}$ in one space d,mension
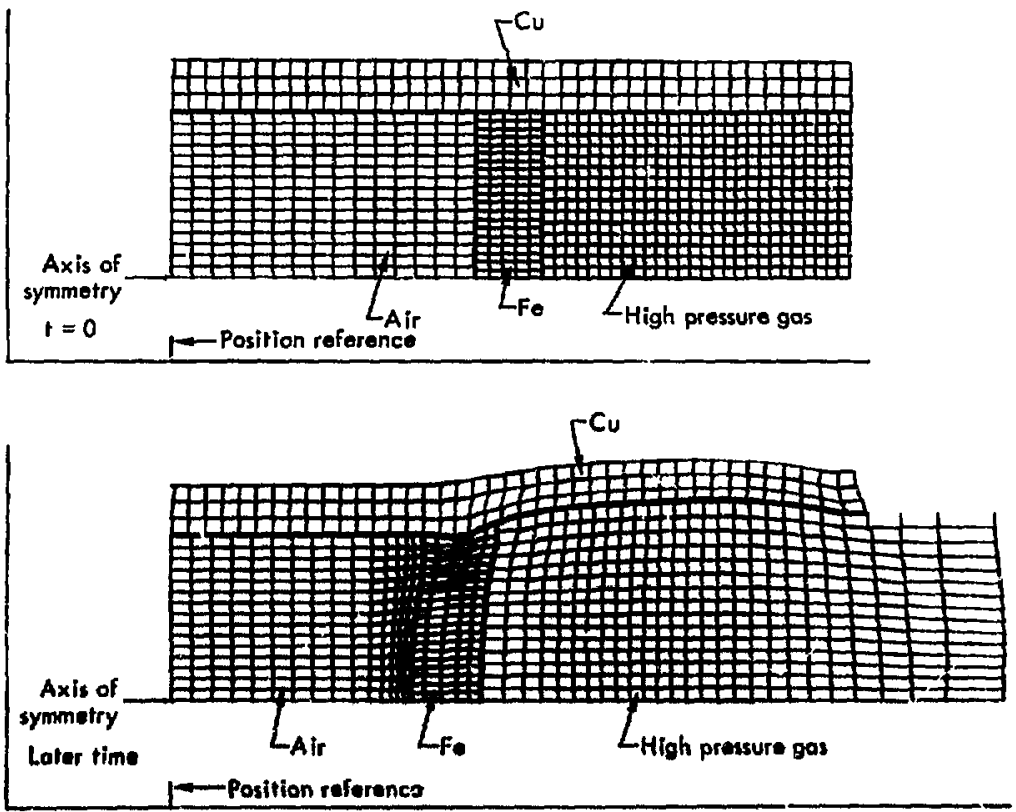

Hig. 9. App.rcation of allide lines in Iwo directions. The calcutation permite sliding between exch pali of wjacent materialt. 
can be checked by a problem sumgested by W. Schultz of $L^{*}$.L. A material with the special properties

$K \equiv 0 . P \equiv 0, c_{v}=\rho_{0} T$ and $\lambda=\frac{10^{6} T^{2}}{i_{R} \rho_{0}}$ is considered, An extreme density, $p_{0}=10^{5} \mathrm{Mg} / \mathrm{m}^{3}$, is used to insure that the radiation energy density is negligible compared to the miterial energy density.

Specializing to one space dimension, the energy equation (7) with the above material model call be written:

$$
\frac{\mathrm{dE}}{\mathrm{dt}}=\mathrm{V}\left[\frac{\partial}{\partial \mathrm{X}}\left(\frac{1}{3}{ }^{\mathrm{a}} \mathrm{R}^{\mathrm{c} \lambda}\right) \frac{\partial T^{4}}{\partial \mathrm{X}}\right]
$$

Substituting for $\lambda, c_{v}$ and $\rho_{0}$ where $E=c_{v} T=\rho_{0} T^{2}$, $V=I$ and $\mathrm{c}=300 \mathrm{Mm} / \mathrm{s}$ givcs:

$$
\frac{d T^{2}}{d t}=\frac{\partial}{\partial X}\left(T^{2} \frac{\partial T^{4}}{\partial X}\right)
$$

This equation has $T^{4}=1-X$ as a solution.

A coiculation was done using the zoning shown in Fig. 10 with boundary conditions $T^{4}=t$ at the left end and $T=0$ at the right and.

The results of the calculation are shown in Fig. 11. The calculated quantity, $T^{4}$, is plotted at zonc centers.

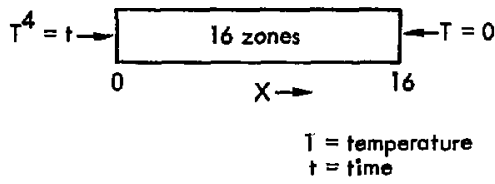

Fig. 10. Calculational grid to test $\mathrm{T}^{4}$ diffusion.

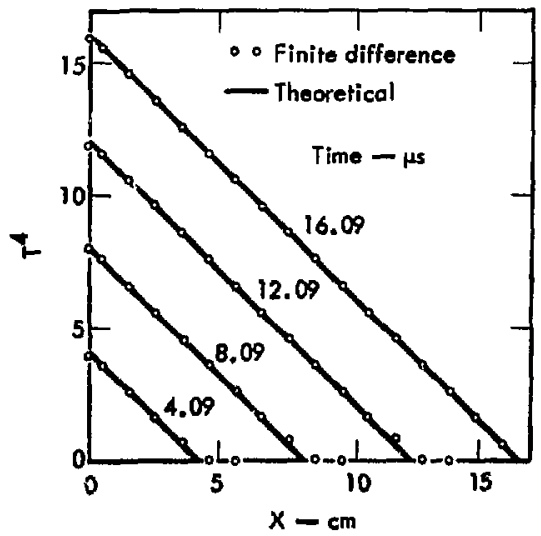

Fig. II. Compatson of the findte difforence calculation with a theoretical calculation for the diffusion of $T$ ". 
It can be seen that all points of the calculated results fall on the straight lines of the theoretical solution, $T^{4}=t-X$.

$A$ lest of the accuracy for solving the thermal diffusion equations in two space dimensions and time un bc obtained by imposing the first Fourier harmonic to the generul solution of the hent equations on a unit scyuare, as an initial condition. The theoretical result for this initial condition is an exponential decay in temperat ure $\mathbf{T}$ as tine $\mathbf{t}$ progresses. Matcrial properties are assumed such that the energy equation, Eq. (7), beconles:

$$
\frac{\mathrm{dT}}{\mathrm{dt}}=\nabla^{2} \mathrm{~T}
$$

A calculation was done using a unit square with a $20 \times 20$ grid. Initial conditions:

$$
T(X, Y, 0)=\sin n X \sin n Y .
$$

Boundary conditions on all four boundaries: $T=0$ for all time.

The theoretical solution to this problem is

$$
T(X, Y, t)=\sin \pi X \sin \pi Y e^{-2 \pi^{2}}
$$

Figure 12 a, b, r shows temperature distribution for various times. Figure $12 \mathrm{~d}$ compares the calculaled and theoretical solutions.

A check of the program for talculnting thermal diffusion with different grids is shown in Fig- 13. The initial conditions are $T=200^{\circ}$ for the spherical region and $T=100^{\circ}$ for the tegion outside the sphere. Reflection boundary conditions are used for all four boundarics. It is scen that the calculated temperaturc contours at $t=1.02 \mu$ are essentially the sume.

\section{Acknowledgments}

The diffusion equations were originally programmed by R. P. Dickinson, Jr. Dick Giroux and Fred Foldvary developed the overall program. Fred Foldvary programmed the logic for diffusion across sliding interfaces. Check problems were done by Jonathon Kutz and Paul Grantham. The assistance of R. Barton and $\mathbf{R}$. Harding in locating errors in the program is gratefully acknowledged. 

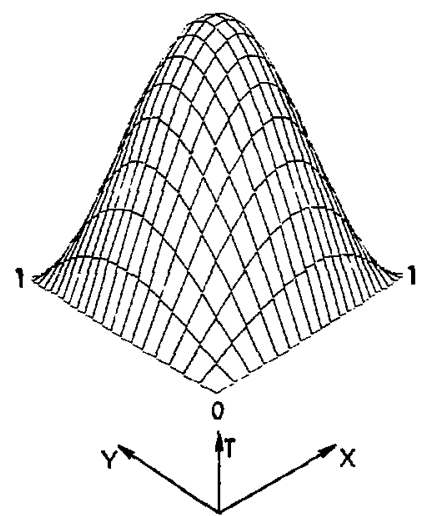

(a)

$$
t=0
$$

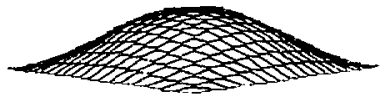

$t=0.075$

\section{(c)}

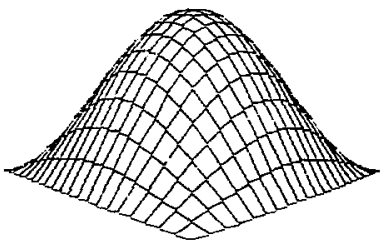

$$
t=0.025
$$

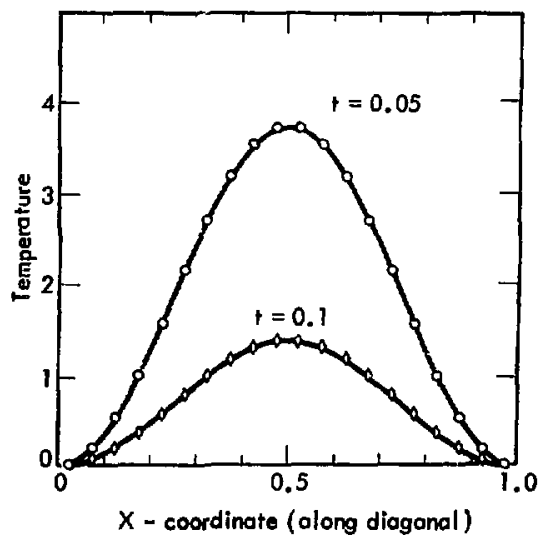

(d)

Fig. 12. Calculation of thermal diffusion in two space dimensions and time.

$a, b$, and c) Temperature distributlons at $1=0$ and al later times

d) Comparisons between the finite difference calculation (points) and the theoretical solution (curves).

Temperature is thown us a function of distunce $(X)$ slong a diagonal of the square. 

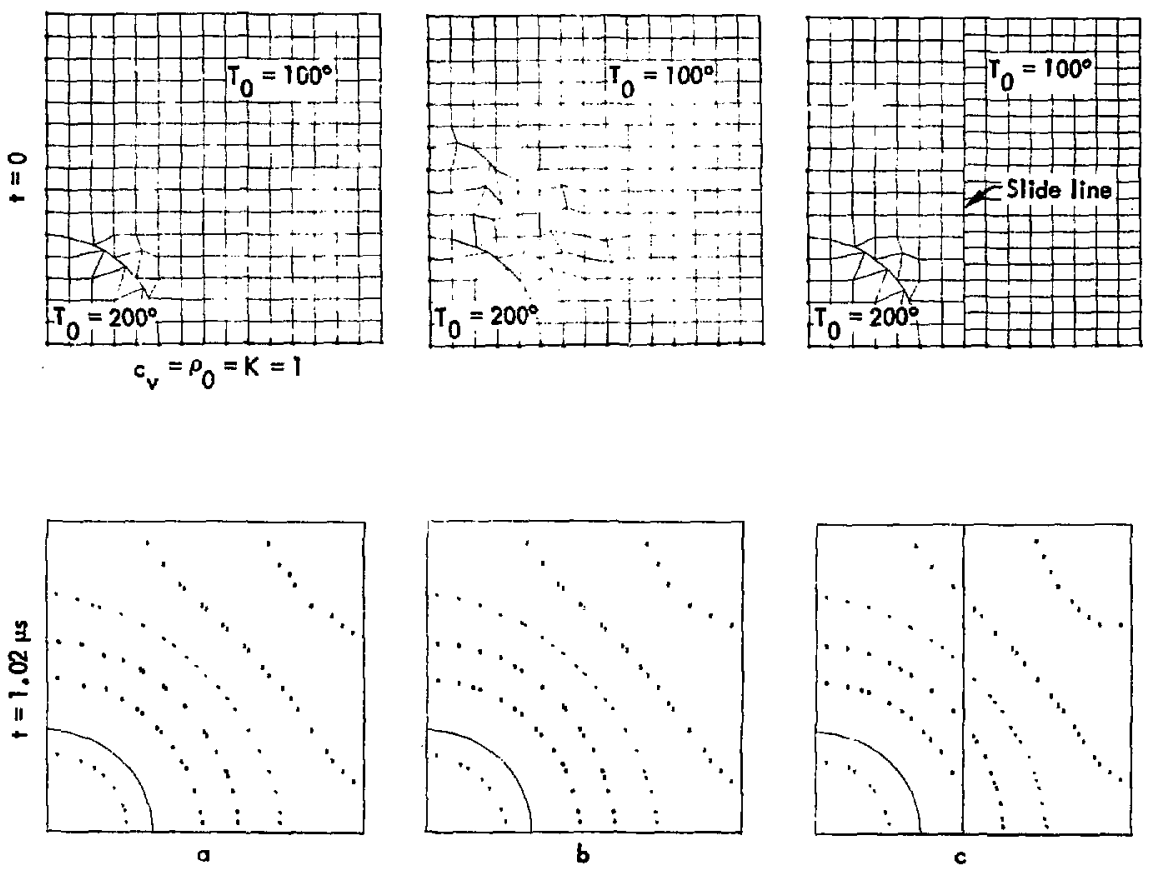

Fig. 13. Calculated temperature contours at $t=1.02 \mu$ s for three calculational grids.
a) Undistorted grid
b) Distorted gridd
c) Grid with side linc. 


\section{References}

1. M. L. Wilkins, Calculation of Elastic-Plastic Flow, Lawrence Live! Inc a I aboratory, Rept. UCRL-7322. Rev. I (1969).

2. R. D. Richtmyer, Difference Methods for Initial Value Problems. (Intersciencz Publishers, InL. . 1957).

3. J. M. LeBlanc, Lawrence Livermore Laboratory, private communicatioli, Jine, 1972.

4. J. Chang, An Introduction to Extrapolation Methods for Numericai Solution of Differential Equations Lawrence Ljvermore Laboratory, Rept. UCID-15992 (ts . 2), 


\section{APPENDIX A}

\section{MAGNETIC FLUX CALCULATION}

\section{Calctlation Scheme for k-Sweep}

First we calculate $\nabla \times \vec{H}$ to be used for the flux change in the $k$-direction. Subscripts in the equations refer to noies and zones indieated in Fig. A-1.

$$
\begin{aligned}
& \nabla \times \vec{H}=\left(\frac{\partial H}{\partial Y}+\frac{H}{Y}\right) \vec{i}-\frac{\partial H}{\partial X} \vec{j} \quad \text { Note: Any quantity shown without a time index is centered at } \\
& \text { a. }\left.\frac{\partial H}{\partial Y}\right|_{1} ^{k}=+\frac{1}{2 A_{1}^{n+1 / 2}}\left[H_{0}^{n+1}\left(X_{2}-X_{4}\right)+H_{0}^{n}\left(X_{4}-X_{12}\right)+H_{\oplus}^{n}\left(X_{12}-X_{5}\right)+H_{0}^{n+1}\left(X_{5}-X_{2}\right)\right] \\
& \left.\frac{H}{\bar{Y}}\right|_{1} ^{k}=+\frac{(H A)_{0}^{n+1}+(H A)_{0}^{n}+(H A)_{0}^{n}+(H A)_{0}^{n+1}}{4 \bar{Y}_{1} A_{1}^{n+1 / 2}} \\
& \left.\frac{\partial H}{\partial X}\right|_{1} ^{k}=-\frac{1}{2 A_{1}^{n+1 / 2}}\left[H_{O}^{n+1}\left(Y_{2}-Y_{4}\right)+H_{\Theta}^{n}\left(Y_{4}-Y_{12}\right)+H_{O}^{n}\left(Y_{12}-Y_{5}\right)+H_{O}^{n+1}\left(Y_{5}-Y_{2}\right)\right] \\
& a_{1}^{k} \equiv\left(\frac{\partial H}{\partial Y}+\frac{H}{Y}\right)_{l}^{k} ; b_{1}^{k} \equiv-\left(\frac{\partial H}{\partial X}\right)_{1}^{k} \\
& A_{1}^{n+1 / 2}=\frac{1}{4}\left(A_{0}^{n+1 / 2}+A_{0}^{n+1 / 2}+A_{O}^{n+1 / 2}+A_{0}^{n+1 / 2}\right) ; \bar{Y}_{1}=\frac{1}{4}\left(Y_{\Theta}^{n+1 / 2}+Y_{0}^{n+1 / 2}+Y_{0}^{n+1 / 2}+Y_{O}^{n+1 / 2}\right)
\end{aligned}
$$

$Y_{\mathscr{G}}^{n+1 / 2}$.etc, as calculated in HEMP. (If $k$ is the $X-a x i s, Y_{\mathscr{O}}, Y_{\Theta}, A_{0}$, and $A_{\oplus}$ are zero.)

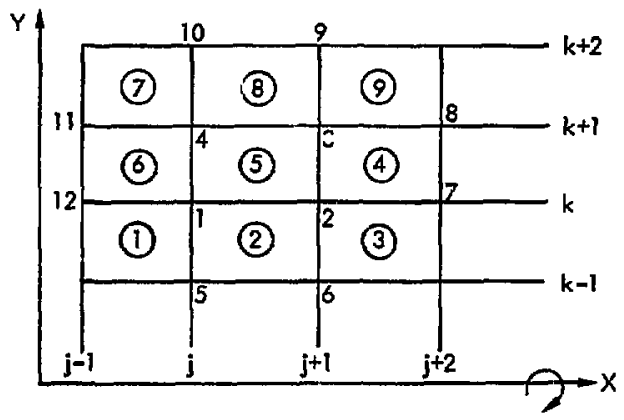

Fig. A.1. Calculational grid for $k$ sweep. Plain integers designate a quantity at a node (j-k line intersection). Integers in circles designate a quantity in the zone center. 
$61^{\circ}$

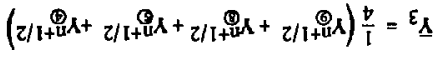

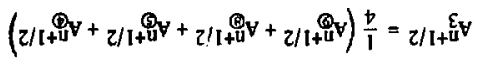

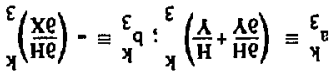

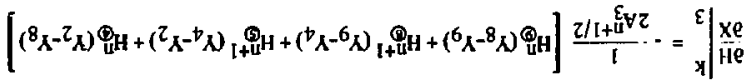

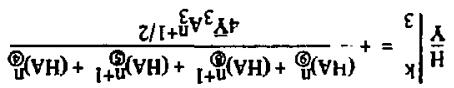

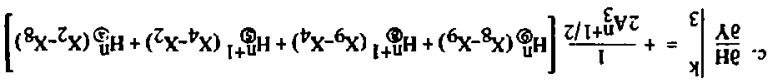

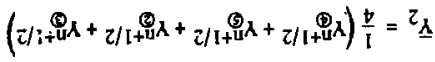

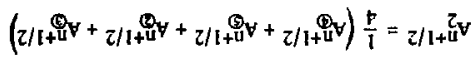

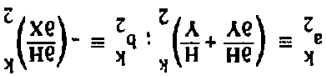

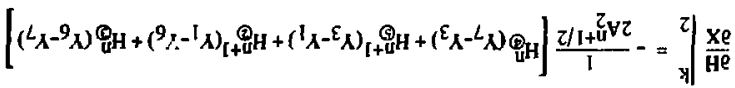

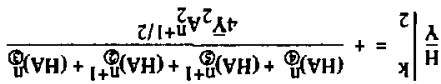

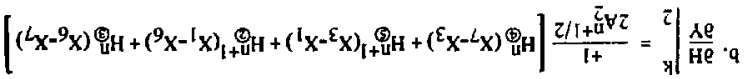




$$
\begin{aligned}
& \mathrm{d}:\left.\quad \frac{\partial \mathrm{H}}{\partial \mathrm{Y}}\right|_{4} ^{\mathrm{k}}=+\frac{1}{2 \mathrm{~A}_{4}^{\mathrm{n}+1 / 2}}\left[\mathrm{H}^{\mathrm{n}+1}\left(\mathrm{X}_{3}-\mathrm{X}_{10}\right)+\mathrm{H}_{0}^{\mathrm{n}}\left(\mathrm{X}_{10}-\mathrm{X}_{11}\right)+\mathrm{H}_{6}^{\mathrm{n}}\left(\mathrm{X}_{11}-\mathrm{X}_{1}\right)+\mathrm{H}_{0}^{\mathrm{n}+1}\left(\mathrm{X}_{1}-\mathrm{X}_{3}\right)\right] \\
& \left.\frac{\mathrm{H}}{\overline{\mathrm{Y}}}\right|_{4} ^{k}=+\frac{(\mathrm{HA})_{0}^{\mathrm{n}+1}+(\mathrm{HA})_{0}^{\mathrm{n}}+(\mathrm{HA})_{0}^{\mathrm{n}}+(\mathrm{HA})_{\mathrm{G}}^{\mathrm{n}+1}}{4 \overline{\mathrm{Y}}_{4} \mathrm{~A}_{4}^{\mathrm{n}+1 / 2}} \\
& \left.\frac{\partial H}{\partial X}\right|_{4} ^{k}=-\frac{1}{2 A_{4}^{n+1 / 2}}\left[H_{0}^{n+1}\left(Y_{3}-Y_{10}\right)+H_{0}^{n}\left(Y_{10}-Y_{11}\right)+H_{0}^{n}\left(Y_{11}-Y_{1}\right)+H_{0}^{n+1}\left(Y_{1}-Y_{3}\right)\right] \\
& a_{4}^{k} \equiv\left(\frac{\partial H}{\partial Y}+\frac{H}{Y}\right)_{4}^{k} ; b_{4}^{k} \equiv-\left(\frac{\partial H}{\partial X}\right)_{4}^{k} \\
& A_{4}^{n+1 / 2}=\frac{1}{4}\left(A_{0}^{n+1 / 2}+A_{0}^{n+1 / 2}+A_{0}^{n+1 / 2}+A_{0}^{n+1 / 2}\right) \\
& \bar{Y}_{4}=\frac{1}{4}\left(Y_{0}^{n+1 / 2}+Y_{O}^{n+1 / 2}+Y_{0}^{n+1 / 2}+Y_{0}^{n+1 / 2}\right)
\end{aligned}
$$

Having computed $\nabla \times \vec{H}$ we now can calculate the change in flux in the $k$ direction, (ie. across $1-2$ and $3-4$ in Fig. A-2).

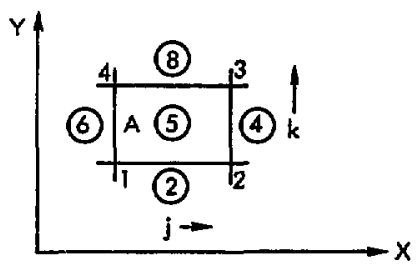

Fig. A-2. Calculational Grid.

$$
\begin{aligned}
4 \pi \mu_{m} \frac{d \Phi}{d t} & =-\nabla \times\left(\frac{1}{c} \nabla \times \vec{H}\right) A \\
\nabla \times \vec{H} & =a \vec{i}+b \vec{j} \\
a & =\left(\frac{\partial H}{\partial Y}+\frac{H}{Y}\right) \\
b & =-\left(\frac{\partial H}{\partial X}\right) \\
c & =\text { electrical conductivity } \\
A & =\text { zone area } \\
4 \pi \mu_{m} \frac{d \Phi}{d t} & =-A_{O}\left(\frac{1}{c} \frac{\partial b}{\partial X}-\frac{1}{C} \frac{\partial a}{\partial Y}\right) \vec{\theta}
\end{aligned}
$$

.20. 
where

$$
\frac{1}{c} \frac{\partial b}{\partial X}=-\frac{1}{2 A_{15}}\left[\frac{1}{c_{1.2}}\left(b_{1}^{k}+b_{2}^{k}\right)\left(Y_{1}-Y_{2}\right)+\frac{1}{c_{3.4}}\left(b_{3}^{k}+b_{4}^{k}\right)\left(Y_{3}-Y_{4}\right)\right]
$$

and

$$
\frac{1}{c} \frac{\partial a}{\partial Y}=+\frac{1}{2 A_{(5)}}\left[\frac{1}{c_{1-2}}\left(a_{1}^{k}+a_{2}^{k}\right)\left(x_{1}-x_{2}\right)+\frac{1}{c_{3-4}}\left(a_{3}^{k}+a_{4}^{k}\right)\left(x_{3}-x_{4}\right)\right] .
$$

Therefore

$$
\begin{aligned}
\left.\Delta \pi \mu_{m} \frac{\left[H^{n+1} A^{n+1}-H^{n} A^{n}\right]_{9}}{\Delta t^{n+1 / 2}}\right|^{k}= & +\frac{1}{2}\left[\frac{1}{C_{1-2}}\left(b_{1}^{k}+b_{2}^{k}\right)\left(Y_{1}-Y_{2}\right)+\frac{1}{C_{3-4}}\left(b_{3}^{k}+b_{4}^{k}\right)\left(Y_{3}-Y_{4}\right)\right. \\
& \left.+\frac{1}{C_{1-2}}\left(a_{1}^{k}+a_{2}^{k}\right)\left(X_{1}-X_{2}\right)+\frac{1}{C_{3-4}}\left(a_{3}^{k}+a_{4}^{k}\right)\left(X_{3}-X_{4}\right)\right]
\end{aligned}
$$

where

$$
c_{1-2}=\frac{\left(c^{n} v^{n+1 / 2} \frac{M}{\rho_{0}}\right)_{\odot}+\left(c^{n} v^{n+1 / 2} \frac{M}{\rho_{0}}\right)_{\odot}}{\left(v^{n+1 / 2} \frac{M}{\rho_{0}}\right)_{\odot}+\left(v^{n+1 / 2} \frac{M}{\rho_{0}}\right)_{\odot}}
$$

and

$$
c_{3-4}=\frac{\left(c^{n} v^{n+1 / 2} \frac{M}{\rho_{0}}\right)_{0}+\left(c^{n} v^{n+1 / 2} \frac{M}{\rho_{0}}\right)_{\odot}}{\left(v^{n+1 / 2} \frac{M}{\rho_{0}}\right)_{0}+\left(v^{n+1 / 2} \frac{M}{\rho_{0}}\right)}
$$

Rewriting the above, we have:

$$
\begin{aligned}
& \left.4 \pi \mu_{m} \frac{\left(H^{n+1} A^{n+1}-H^{n} A^{n}\right)}{\Delta t^{n+1 / 2}}\right|^{k} \\
& =\frac{1}{4} H_{G}^{n+1}\left[\left(\frac{Y_{2}-Y_{4}}{A_{1}}+\frac{Y_{3}-Y_{1}}{A_{2}}\right)\left(\frac{Y_{1}-Y_{2}}{C_{1-2}}\right)+\left(\frac{Y_{4}-Y_{3}}{A_{3}}+\frac{Y_{1}-Y_{3}}{A_{4}}\right)\left(\frac{Y_{3}-Y_{4}}{C_{3-4}}\right)\right. \\
& +\left(\frac{X_{2}-X_{4}}{A_{1}}+\frac{X_{3}-X_{1}}{A_{2}}+\frac{A_{3}^{n+1}}{2 \bar{Y}_{1} A_{1}}+\frac{A_{9}^{n+1}}{2 \bar{Y}_{2} A_{2}}\right)\left(\frac{X_{1}-X_{2}}{C_{1-2}}\right) \\
& \left.+\left(\frac{X_{4}-X_{3}}{A_{3}}+\frac{X_{1}-X_{3}}{A_{4}}+\frac{A_{3}^{n+1}}{2 \bar{Y}_{3} A_{3}}+\frac{A_{(3)}^{n+1}}{2 \bar{Y}_{4} A_{4}}\right)\left(\frac{X_{3}-X_{4}}{C_{3-4}}\right)\right]
\end{aligned}
$$

-21- 


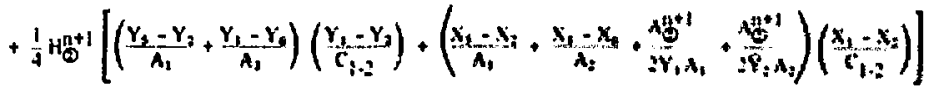

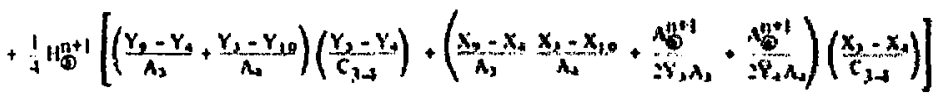

$$
\begin{aligned}
& +\frac{1}{4} u_{0}^{n}\left[\left(\frac{Y_{1}-Y_{12}}{\Lambda_{1}}\right)\left(\frac{Y_{1}-Y_{2}}{C_{1.2}}\right)+\left(\frac{Y_{11}-Y_{2}}{\lambda_{1}}\right)\left(\frac{Y_{1}-Y_{1}}{r_{3+1}}\right)\right.
\end{aligned}
$$

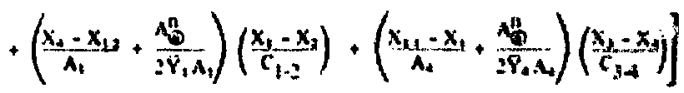

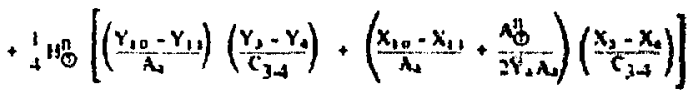

$$
\begin{aligned}
& +\frac{1}{4} 11_{0}^{n}\left[\left(\frac{Y_{12}-Y_{3}}{A_{1}}\right)\left(\frac{Y_{1}-Y_{2}}{C_{1.2}}\right)+\left(\frac{X_{12}-X_{3}}{A_{1}}+\frac{A_{0}^{n}}{2 Y_{1} A_{1}}\right)\left(\frac{\left.X_{1}-X_{2}\right)}{C_{1.2}}\right)\right] \\
& +\frac{1}{4} H_{\Theta}^{n}\left[\left(\frac{Y_{9}-Y_{3}}{A_{3}}\right)\left(\frac{Y_{1}-Y_{2}}{C_{1 \cdot 2}}\right)+\left(\frac{Y_{3}-Y_{2}}{A_{3}}\right)\left(\frac{Y_{3}-Y_{4}}{C_{3-1}}\right)\right. \\
& \left.+\left(\frac{x_{2}-x_{3}}{A_{2}}+\frac{A_{0}^{n}}{2 Y_{2} A_{2}}\right)\left(\frac{x_{1}-x_{2}}{C_{1-2}}\right)+\left(\frac{x_{3}-x_{3}}{A_{3}}+\frac{A_{0}^{n}}{2 \hat{Y}_{2} A_{3}}\right)\left(\frac{x_{3}-x_{1}}{C_{3-4}}\right)\right] \\
& +\frac{1}{4} H_{0}^{n}\left[\left(\frac{Y_{0}-Y_{2}}{A_{2}}\right)\left(\frac{Y_{1}-Y_{2}}{C_{1.2}}\right)+\left(\frac{X_{6}-X_{1}}{A_{2}}+\frac{A_{0}^{n}}{2 Y_{2} A_{2}}\right)\left(\frac{X_{1}-X_{2}}{C_{1-2}}\right)\right] \\
& +\frac{1}{4} H_{\mathscr{Q}}^{\mathrm{n}}\left[\left(\frac{Y_{3}-Y_{9}}{A_{3}}\right)\left(\frac{Y_{3}-Y_{4}}{C_{3-4}}\right)+\left(\frac{X_{3}-X_{9}}{A_{3}}+\frac{A_{0}^{n}}{2 Y_{3} A_{3}}\right)\left(\frac{X_{3}-X_{4}}{C_{3-4}}\right)\right] .
\end{aligned}
$$

Now we arrange the equations for $\mathbf{H}^{\mathrm{n}+1}$ into a group of linear equations. Referring to Fig. A-3. rewrite Eq. (A.J). which expresses the change in flux in the $k$-direction of zone $j+1 / 2, k+1 / 2$ (zone $(D)$ ), as a linear equation in $\mathbf{H}^{\mathbf{n + 1}}$.

.22. 


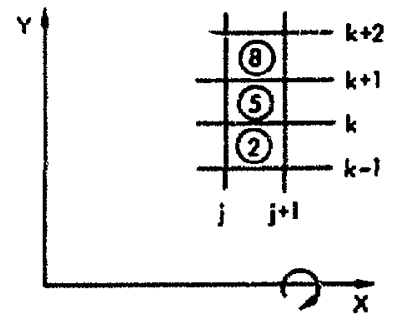

Fit. A-3. Crid for calculating Uus ctange in zone D from contributions in the k-ditection.

$$
\begin{aligned}
& A_{i} H_{j+1}+B_{j} H_{j}+C_{i} H_{i-j}=D_{i} \\
& H_{i+1} \text { 로 } H^{n+1}=1_{j+1 / 2 k+3 / 2}^{n+1} \\
& n_{1} \equiv 100+1=10+12 k+1 / 2 \\
& \left\|H_{i-1} \equiv 1\right\|^{+1}=n_{j+1 / 2 k-1 / 2}^{n+1} \\
& A_{1}=-\frac{1}{4} \frac{A_{1}^{n+1 / 2}}{4 \pi \mu_{m}}\left[\left(\frac{Y_{2}-Y_{4}}{A_{3}}+\frac{Y_{3}-Y_{10}}{A_{4}}\right)\left(\frac{Y_{3}-Y_{4}}{C_{34}}\right)\right. \\
& \left.-\left(\frac{x_{0}-x_{1}}{\lambda_{3}}+\frac{x_{3}-x_{10}}{A_{0}}+\frac{A_{3}^{n+1}}{2 V_{3} A_{3}}+\frac{A_{3}^{n+1}}{2 \gamma_{1} A_{4}}\right)\left(\frac{x_{3}-x_{1}}{C_{3-4}}\right)\right] \\
& B_{1}=A_{0}^{A^{+1}}-\frac{1}{4} \frac{\Delta^{n+1 / 2}}{\Delta \pi \mu_{m}}\left[\left(\frac{Y_{2}-Y_{4}}{A_{1}}+\frac{Y_{3}-Y_{1}}{A_{2}}\right)\left(\frac{Y_{1}-Y_{2}}{C_{1.2}}\right)+\left(\frac{Y_{1}-Y_{2}}{A_{3}}+\frac{Y_{1}-Y_{3}}{\Lambda_{4}}\right)\left(\frac{Y_{3}-Y_{4}}{C_{3-4}}\right)\right. \\
& +\left(\frac{x_{2}-x_{2}}{A_{1}}+\frac{x_{3}-x_{1}}{A_{2}}+\frac{A_{0}^{n+1}}{2 Y_{1} A_{1}}+\frac{A_{0}^{n+1}}{2 \gamma_{2} A_{2}}\right)\left(\frac{x_{1}-x_{2}}{C_{1-2}}\right)
\end{aligned}
$$

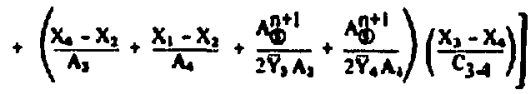




$$
\begin{aligned}
& C_{i}=-\frac{1}{4} \frac{\Delta i^{n+1}}{4 \pi \mu_{m}} \int\left(\frac{Y_{3}-Y_{2}}{A_{1}}+\frac{Y_{1}-Y_{6}}{A_{2}}\right)\left(\frac{Y_{1}-Y_{2}}{C_{1.2}}\right)+\left(\frac{X_{5}-X_{2}}{A_{1}}+\frac{X_{1}-X_{6}}{A_{2}}\right. \\
& \left.\left.+\frac{A_{0}^{n+1}}{2 \bar{Y}_{1} A_{1}}+\frac{A_{\Theta}^{n+1}}{2 \bar{Y}_{2} A_{2}}\right)\left(\frac{x_{1}-X_{2}}{C_{1.2}}\right)\right] \\
& Q_{1}=(H A)_{D}^{n}+\frac{1}{4} \frac{4 t^{n+1 / 2}}{4 \pi \mu_{m}} \int H_{0}^{n}\left[\left(\frac{Y_{4}-Y_{12}}{A_{1}}\right)\left(\frac{Y_{1}-Y_{2}}{C_{1-2}}\right)+\left(\frac{Y_{11}-Y_{1}}{A_{4}}\right)\left(\frac{Y_{3}-Y_{4}}{C_{34}}\right)\right. \\
& \left.+\left(\frac{x_{4}}{A_{1}}-x_{12}+\frac{A_{0}^{n}}{2 \bar{Y}_{1} A_{1}}\right)\left(\frac{X_{1}-x_{3}}{C_{1.2}}\right)+\left(\frac{x_{11}-X_{1}}{A_{4}}+\frac{A_{6}^{n}}{2 \bar{Y}_{4} A_{4}}\right)\left(\frac{X_{3}-X_{4}}{C_{3-4}}\right)\right] \\
& +H_{O}\left[\left(\frac{Y_{10}-Y_{11}}{A_{4}}\right)\left(\frac{Y_{3}-Y_{4}}{C_{34}}\right)+\left(\frac{X_{10}-X_{11}}{A_{4}}+\frac{A_{O}^{\prime \prime}}{2 \bar{Y}_{4} A_{4}}\right)\left(\frac{X_{3}-X_{4}}{C_{3-4}}\right)\right] \\
& +1 \|_{\mathbb{D}}^{n}\left[\left(\frac{Y_{12}-Y_{5}}{A_{1}}\right)\left(\frac{Y_{1}-Y_{2}}{C_{1-2}}\right)+\left(\frac{X_{12}-X_{3}}{A_{1}}+\frac{A_{0}^{n}}{2 Y_{1} A_{1}}\right)\left(\frac{X_{1}-X_{2}}{C_{1-2}}\right)\right] \\
& +H_{0}\left[\left(\frac{Y_{1}-Y_{3}}{A_{2}}\right)\left(\frac{Y_{1}-Y_{2}}{C_{1.2}}\right)+\left(\frac{Y_{2}-Y_{8}}{A_{3}}\right)\left(\frac{Y_{3}-Y_{4}}{C_{3-4}}\right)\right. \\
& \left.+\left(\frac{X_{2}-X_{3}}{A_{2}}+\frac{A_{\Theta}^{n}}{2 \bar{Y}_{2} A_{2}}\right)\left(\frac{X_{1}-X_{2}}{C_{1 \cdot 2}}\right)+\left(\frac{X_{2}-X_{8}}{A_{3}}+\frac{A_{\mathcal{O}}^{n}}{2 \bar{Y}_{3} A_{3}}\right)\left(\frac{X_{3}-X_{4}}{C_{34}}\right)\right] \\
& +H_{0}^{n}\left[\left(\frac{Y_{6}-Y_{7}}{A_{2}}\right)\left(\frac{Y_{1}-Y_{2}}{C_{1.2}}\right)+\left(\frac{X_{6}-X_{7}}{A_{2}}+\frac{A_{3}^{n}}{2 Y_{2} A_{2}}\right)\left(\frac{X_{1}-X_{2}}{C_{1-2}}\right)\right] \\
& \left.+H_{O}^{n}\left[\left(\frac{Y_{5}-Y_{9}}{A_{3}}\right)\left(\frac{Y_{3}-Y_{4}}{C_{3-4}}\right)+\left(\frac{X_{B}-X_{9}}{A_{3}}+\frac{A_{0}^{n}}{2 \bar{Y}_{3} A_{3}}\right)\left(\frac{X_{3}-X_{4}}{C_{3-4}}\right)\right]\right\}
\end{aligned}
$$

The unethod of solution for a set of linear equations of this form is given in Appendix $C$. 


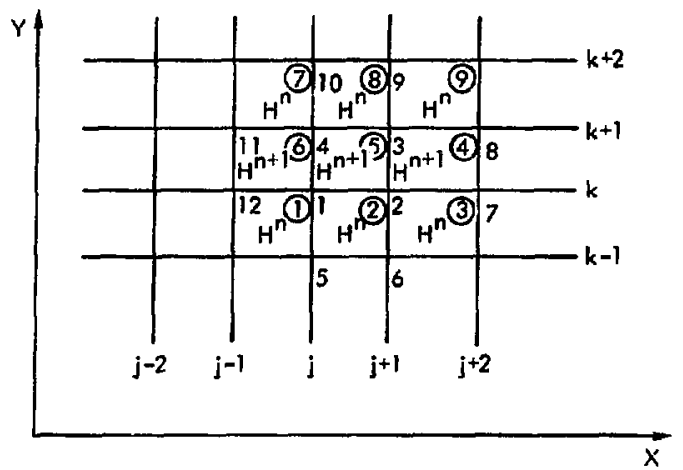

Fig. A-4. Calculational grid for j-sweep.

Next, referring to Fig. A 4 for the numbering of nodes, zones, and values of $H$, ve execute in analoguas calculation of $\nabla \times \overrightarrow{\mathbf{H}}$ to be used for the flux change in the $\mathrm{j}$-direction.

$$
\begin{aligned}
& \nabla \times \vec{H}=\left(\frac{\partial H}{\partial \bar{Y}}+\frac{H}{Y}\right) \vec{i}-\frac{\partial H}{\partial X} \vec{j} \\
& \text { a. }\left.\quad \frac{\partial H}{\partial Y}\right|_{1} ^{j}=+\frac{1}{2 A_{1}^{n+1 / 2}}\left[H_{\Theta}^{n+1}\left(X_{2}-X_{4}\right)+H_{\Theta}^{n+1}\left(X_{4}-X_{12}\right)+H_{\Theta}^{n}\left(X_{12}-X_{4}\right)+H_{\Theta}^{n}\left(X_{5}-X_{2}\right)\right] \\
& \left.\frac{H}{\bar{Y}}\right|_{1} ^{j}=+\frac{(H A)_{\mathscr{G}}^{\Pi+1}+(H A)_{\odot}^{n+1}+(H A)_{D}^{n}+(H A)_{\odot}^{n}}{4 \bar{Y}_{1} A_{1}^{n+i / 2}} \\
& \left.\frac{\partial H}{\partial X}\right|_{1} ^{j}=-\frac{1}{2 A_{1}{ }^{n+1 / 2}}\left[H_{0}^{n+1}\left(Y_{2}-Y_{4}\right)+H_{0}^{n+1}\left(Y_{4}-Y_{12}\right)+H_{0}^{\pi}\left(Y_{12}-Y_{5}\right)+H_{(2)}^{n}\left(Y_{5}-Y_{2}\right)\right] \\
& \underset{\mathbf{j}}{\mathbf{j}} \equiv\left(\frac{\partial \mathrm{H}}{\partial \bar{Y}}+\frac{\mathrm{H}}{\mathrm{Y}}\right)_{1}^{\mathbf{j}} ; \mathbf{b}_{1}^{\mathbf{j}} \equiv-\left(\frac{\partial H}{\partial X}\right)_{1}^{\mathfrak{j}}
\end{aligned}
$$




$$
\begin{aligned}
& A_{1}^{n+1 / 2}=\frac{1}{+}\left(A_{0}^{n+2}+A_{0}^{n+12}+A_{0}^{n+1 / 2}+A_{0}^{n+1 / 2}\right) \\
& \gamma_{1}=\frac{1}{4}\left(n_{0}^{n+1 / 2}+v_{0}^{n+1 / 2}+v_{0}^{n+1 / 2}+v_{0}^{n+1 / 2}\right)
\end{aligned}
$$

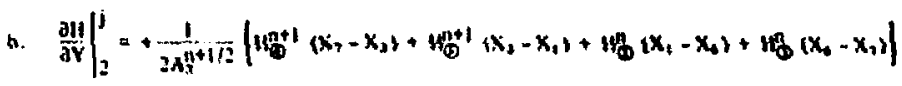

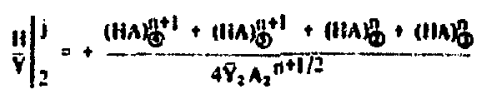$$
\left.\frac{\partial H}{\partial s^{j}}\right|_{2} ^{j}=-\frac{1}{2 A_{2}^{n+1 / 2}}\left|H_{0}^{n+1}\left(Y_{2}-Y_{3}\right)+H_{g}^{+1}\left(Y_{2}-Y_{1}\right)+H_{0}\left(Y_{1}-Y_{0}\right)+H_{0}\left(Y_{0}-Y_{7}\right)\right|
$$$$
\hat{z}_{2}=\left(\frac{\partial H}{\partial \bar{Y}}+\frac{H}{\bar{Y}}\right)_{2}^{j}: b_{2}^{j}=-\left(\frac{\partial H}{\partial X}\right)_{2}^{j}
$$$$
A_{2}^{n+1 / 2}=\frac{1}{4}\left(A_{0}^{n+1 / 2}+A_{0}^{+1 / 2}+A_{0}^{n+1 / 2}+A_{D}^{n+1 / 2}\right)
$$$$
\bar{Y}=\frac{1}{4}\left(Y_{0}^{n+1 / 2}+Y_{0}^{n+1 / 2}+Y_{0}^{n+1 / 2}+Y_{0}^{n+1 / 2}\right)
$$

c. $\left.\quad \frac{\partial H}{\partial Y}\right|_{3} ^{j}=+\frac{1}{2 A_{3}^{n+1 / 2}}\left[H_{\Theta}^{n}\left(X_{1}-X_{0}\right)+H_{0}^{n}\left(X_{9}-X_{A}\right)+H_{\Theta}^{n+1}\left(X_{1}-X_{2}\right)+H_{\Theta}^{n^{+1}}\left(X_{2}-X_{8}\right)\right]$

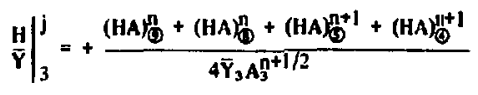

$$
\begin{aligned}
& \left.\frac{\partial H}{\partial X}\right|_{3} ^{j}=-\frac{1}{2 A_{3}^{n+1 / 2}}\left[H_{\oplus}^{n}\left(Y_{8}-Y_{9}\right)+H_{\oplus}^{n}\left(Y_{8}-Y_{4}\right)+H_{\oplus}^{n+1}\left(Y_{4}-Y_{2}\right)+H_{\odot}^{n+i}\left(Y_{2}-Y_{8}\right)\right] \\
& a_{3}^{j}=\left(\frac{\partial H}{\partial Y}+\frac{H}{Y}\right)_{3}^{j}: b_{3}^{j}=-\left(\frac{\partial H}{\partial X}\right)_{3}^{j}
\end{aligned}
$$

20 . 


$$
\begin{aligned}
& A_{2}^{n+1 / 2}=\frac{1}{4}\left(\lambda_{0}^{n+1 / 2}+A_{0}^{n+1 / 2}+A_{0}^{n+1 / 2}+\lambda_{0}^{n+1 / 2}\right) \\
& r_{3}=\frac{1}{4}\left(y_{0}^{11+1 / 2}+v_{0}^{11+1 / 2}+v_{0}^{11112}, 8_{0}^{n+1 / 2}\right)
\end{aligned}
$$

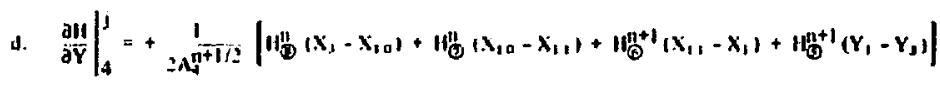

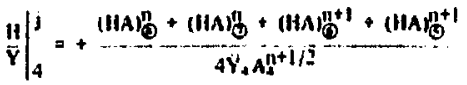

$$
\begin{aligned}
& \left.\frac{\partial H}{\partial X}\right|_{4} ^{j}=-\frac{1}{2 A^{n+1} / 2}\left[H_{O}^{D}\left(Y_{3}-Y_{10}\right)+H_{O}^{n}\left(Y_{10}-Y_{11}\right)+H_{O}^{n+1}\left(Y_{11}-Y_{1}\right)+H_{\Theta}^{n+1}\left(Y_{1}-Y_{3}\right)\right] \\
& \mathrm{a}_{4}^{\mathrm{j}}=\left(\frac{\partial H}{\partial \bar{Y}}+\frac{H}{\bar{Y}}\right)_{4}^{j}: b_{4}^{j}=-\left(\frac{\partial H}{\partial X}\right)_{4}^{j} \\
& A_{4}^{n+1 / 2}=\frac{1}{4}\left(A_{0}^{n+1 / 2}+A_{b}^{n+1 / 2}+A_{0}^{n+1 / 2}+A_{0}^{n+1 / 2}\right) \\
& \vec{Y}_{4}=\frac{1}{4}\left(Y_{0}^{n+1 / 2}+Y_{0}^{n+1 / 2}+Y_{0}^{n+1 / 2}+Y_{0}^{n+1 / 2}\right)
\end{aligned}
$$

Using this value of $\nabla \times \vec{H}$ we calculate the change in flux in the j-direction. ie. across $2 \cdot 3$ and $4-1$ in Fig. A.5.

$$
\begin{aligned}
& 4 \pi \mu_{m} \frac{d \phi}{d t}=-\nabla \times\left(\frac{1}{C} \nabla \times \vec{H}\right) A \\
& \nabla \times \vec{H}=a \vec{i}+b \vec{j}
\end{aligned}
$$

$-27$. 


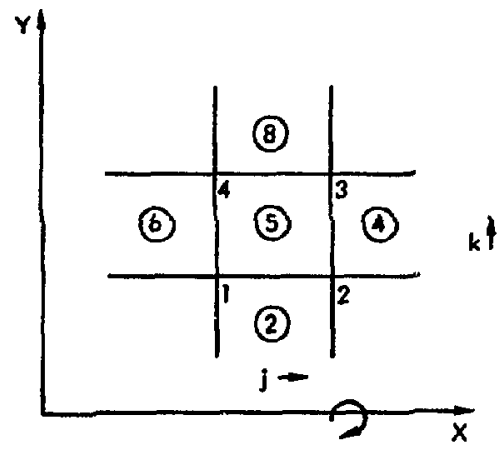

Fig. A-5. Calculational grid.

$$
\begin{aligned}
& a=\left(\frac{\partial H}{\partial Y}+\frac{H}{Y}\right) \\
& b=-\left(\frac{\partial H}{\partial X}\right) \\
& c=\text { electrical conductivity } \\
& 4 \pi \mu_{m} \frac{d \phi}{d t}=-A_{(J)}\left[\frac{1}{C} \frac{\partial b}{\partial X}-\frac{1}{C} \frac{\partial a}{\partial \bar{Y}}\right] \vec{\theta}
\end{aligned}
$$

where

$$
\frac{1}{C} \frac{\partial b}{\partial X}=-\frac{1}{2 A_{0}}\left[\frac{1}{C_{2-3}}\left(b_{2}^{j}+b_{3}^{j}\right)\left(Y_{2}-Y_{3}\right)+\frac{1}{C_{4-1}}\left(b_{4}^{j}+b_{1}^{j}\right)\left(Y_{4}-Y_{1}\right)\right]
$$

and

$$
\frac{1}{C} \frac{\partial a}{\partial Y}=+\frac{1}{2 A_{0}}\left[\frac{1}{C_{2-3}}\left(a_{2}^{j}+a_{3}^{j}\right)\left(X_{2}-X_{9}\right)+\frac{1}{C_{4-1}}\left(a_{4}^{j}+a_{1}^{j}\right)\left(X_{4}-X_{1}\right)\right] .
$$

\section{Therefure}

$$
\begin{aligned}
\frac{\Delta \pi \mu_{m}\left[(H A)^{n+1}-(H A)^{n}\right]_{0}}{\Delta t^{n+1 / 2}=} & +\frac{1}{2}\left[\frac{1}{C_{2-3}}\left(b_{2}^{j}+b_{3}^{j}\right)\left(Y_{2}-Y_{3}\right)+\frac{1}{C_{4-1}}\left(b_{a}^{j}+b_{1}^{j}\right)\left(Y_{4}-Y_{1}\right)\right. \\
& \left.+\frac{1}{C_{2-3}}\left(a_{2}^{j}+a_{3}^{j}\right)\left(X_{2}-X_{3}\right)+\frac{1}{C_{4-1}}\left(a_{4}^{j}+a_{1}^{j}\right)\left(X_{4}-X_{1}\right)\right]
\end{aligned}
$$


where

$$
c_{2 \cdot 3}=\frac{\left(c^{n} v^{n+1 / 2} \frac{N}{\rho_{0}}\right)_{\Theta}+\left(c^{n} v^{n+1 / 2} \frac{M}{\rho_{0}}\right)_{\Theta}}{\left(v^{n+1 / 2} \frac{M}{\rho_{0}}\right)_{\odot}+\left(v^{n+1 / 2} \frac{M}{\rho_{0}}\right)_{\odot}}
$$

and

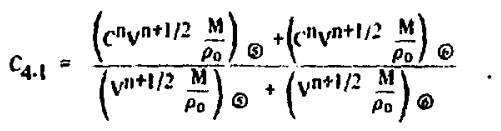

Rewriting the above gives

$$
\begin{aligned}
& \left.4 \pi \mu_{m} \frac{\left[(H A)^{n+1}-(H A)^{n}\right]_{O}}{\Delta i^{n+1 / 2}}\right|^{j} \\
& =\frac{1}{4} H_{00}^{n+1}\left[\left(\frac{Y_{3}-Y_{1}}{A_{2}}+\frac{Y_{4}-Y_{2}}{A_{3}}\right)\left(\frac{Y_{2}-Y_{3}}{C_{2-3}}\right)+\left(\frac{Y_{1}-Y_{3}}{A_{4}}+\frac{Y_{2}-Y_{4}}{A_{3}}\right)\left(\frac{Y_{4}-Y_{3}}{C_{4-1}}\right)\right. \\
& +\left(\frac{X_{3}-X_{1}}{A_{2}}+\frac{X_{4}-X_{2}}{A_{3}}+\frac{A_{3}^{n+1}}{2 \overline{Y_{3} A_{2}}}+\frac{A_{6}^{n+1}}{2 \overline{Y_{3}} A_{3}}\right)\left(\frac{X_{2}-X_{3}}{C_{2-3}}\right) \\
& \left.+\left(\frac{X_{1}-X_{3}}{A_{4}}+\frac{X_{2}-X_{4}}{A_{1}}+\frac{A_{(3)}^{n+1}}{2 \bar{Y}_{1} A_{4}}+\frac{A_{5}^{n+1}}{2 \bar{Y}_{1} A_{1}}\right)\left(\frac{X_{4}-X_{1}}{C_{4-1}}\right)\right] \\
& +\frac{1}{4} H_{\oplus}^{n+1}\left[\left(\frac{Y_{2}-Y_{3}}{A_{2}}+\frac{Y_{2}-Y_{8}}{A_{3}}\right)\left(\frac{Y_{2}-Y_{3}}{C_{2 \cdot 3}}\right)+\left(\frac{X_{1}-X_{3}}{A_{2}}+\frac{X_{2}-X_{8}}{A_{3}}+\frac{A_{\oplus}^{n+1}}{2 \bar{Y}_{2} A_{2}}+\frac{A_{\oplus}^{n+1}}{2 \bar{Y}_{3} A_{3}}\right)\left(\frac{X_{2}-X_{3}}{C_{2 \cdot 3}}\right)\right] \\
& +\frac{1}{4} H_{\oplus}^{n+1}\left[\left(\frac{Y_{11}-Y_{4}}{A_{4}}+\frac{Y_{9}-Y_{12}}{A_{1}}\right)\left(\frac{Y_{4}-Y_{1}}{C_{4-1}}\right)\right. \\
& \left.+\left(\frac{X_{11}-X_{1}}{A_{4}}+\frac{X_{4}-X_{12}}{A_{1}}+\frac{A_{6}^{n+1}}{2 \bar{Y}_{4} A_{4}}+\frac{A_{6}^{\pi+1}}{2 \bar{Y}_{1} A_{1}}\right)\left(\frac{X_{4}-X_{1}}{C_{4-1}}\right)\right] \\
& +\frac{1}{4} H_{0}^{n}\left[\left(\frac{Y_{0}-Y_{4}}{A_{3}}\right)\left(\frac{Y_{2}-Y_{3}}{C_{2 \cdot 3}}\right)+\left(\frac{Y_{3}-Y_{10}}{A_{4}}\right)\left(\frac{Y_{4}-Y_{1}}{C_{4-1}}\right)\right.
\end{aligned}
$$

.29. 


$$
\begin{aligned}
& \left.+\left(\frac{X_{9}-X_{4}}{A_{3}}+\frac{A_{9}^{n}}{2 \bar{Y}_{3} A_{3}}\right)\left(\frac{X_{2}-X_{3}}{C_{2-3}}\right)+\left(\frac{X_{3}-X_{10}}{A_{4}}+\frac{A_{9}^{n}}{2 \bar{Y}_{4} A_{4}}\right)\left(\frac{X_{4}-X_{1}}{C_{4-1}}\right)\right] \\
& +\frac{1}{4} H_{0}^{n}\left[\left(\frac{Y_{1}-Y_{6}}{A_{2}}\right)\left(\frac{Y_{2}-Y_{3}}{C_{2-3}}\right)+\left(\frac{Y_{5}-Y_{2}}{A_{1}}\right)\left(\frac{Y_{4}-Y_{1}}{C_{4-1}}\right)\right. \\
& \left.+\left(\frac{X_{1}-X_{6}}{A_{2}}+\frac{A_{0}^{n}}{2 \bar{Y}_{2} A_{2}}\right)\left(\frac{X_{2}-X_{3}}{C_{2-3}}\right)+\left(\frac{X_{5}-X_{2}}{A_{1}}+\frac{A_{6}^{n}}{2 \bar{Y}_{1} A_{1}}\right)\left(\frac{X_{4}-X_{1}}{C_{4-1}}\right)\right] \\
& +\frac{1}{4} H_{\mathscr{O}}^{n}\left[\left(\frac{Y_{12}-Y_{5}}{A_{1}}\right)\left(\frac{Y_{4}-Y_{1}}{C_{4-1}}\right)+\left(\frac{X_{12}-X_{5}}{A_{1}}+\frac{A_{D}^{n}}{2 \bar{Y}_{1} A_{1}}\right)\left(\frac{X_{4}-X_{1}}{C_{4-1}}\right)\right] \\
& +\frac{1}{4} H_{9}^{n}\left[\left(\frac{Y_{6}-Y_{7}}{A_{2}}\right)\left(\frac{Y_{2}-Y_{3}}{C_{2-3}}\right)+\left(\frac{X_{6}-X_{7}}{A_{2}}+\frac{A_{9}^{n}}{2 \bar{Y}_{2} A_{2}}\right)\left(\frac{X_{2}-X_{3}}{C_{2-3}}\right)\right] \\
& +\frac{1}{4} H_{\odot}^{\mathrm{n}}\left[\left(\frac{Y_{8}-Y_{9}}{A_{3}}\right)\left(\frac{Y_{2}-Y_{3}}{C_{2-3}}\right)+\left(\frac{X_{B}-X_{9}}{A_{3}}+\frac{A_{9}^{n}}{2 \bar{Y}_{3} A_{3}}\right)\left(\frac{X_{2}-X_{3}}{C_{2-3}}\right)\right] \\
& \left.+\frac{1}{4} H_{O}^{n}\left[\left(\frac{Y_{10}-Y_{11}}{A_{4}}\right)\left(\frac{Y_{4}-Y_{1}}{C_{4-1}}\right)+\left(\frac{X_{10}-X_{11}}{A_{4}}+\frac{A_{O}^{n}}{2 \bar{Y}_{4} A_{4}}\right) ; \frac{X_{4}-X_{1}}{C_{4-1}}\right)\right] .
\end{aligned}
$$

Arranging the equation for $\mathrm{H}^{\mathrm{n}+1}$ into a group of linear equations for a given $\mathrm{k}$-line (see Fig. A-6):

$$
\begin{aligned}
& H_{j+1} \equiv H_{(9)}^{n+1}=\underset{j+3 / 2, k+1 / 2}{n+1} \\
& H_{i} \equiv H_{()}^{n+1}=\underset{j+1 / 2, k+1 / 2}{n+1} \\
& H_{i-1} \equiv H_{(6)}^{n+1}=H_{j-1 / 2, k+1 / 2}^{n+1}
\end{aligned}
$$

$-30-$ 


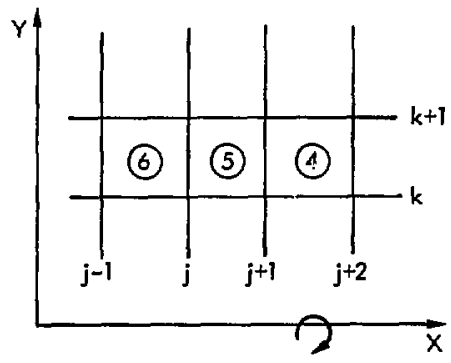

Fig. A-6. Grid for calculatıg, flux change in zone () from contributions in the $\mathrm{j}$-direction.

Newriting again Eq. (A.2), the change in flux in the j-direction

$$
A_{i} H_{i+1}+B_{i} H_{i}+C_{i} H_{i-1}=D_{i} \text {. }
$$

where

$$
\begin{aligned}
A_{j}= & -\frac{1}{4} \frac{\Delta t^{n+1 / 2}}{4 \pi \mu_{m}}\left[\left(\frac{Y_{3}-Y_{3}}{A_{2}}+\frac{Y_{2}-Y_{B}}{A_{3}}\right)\left(\frac{Y_{2}-Y_{3}}{C_{2-3}}\right)+\left(\frac{X_{1}-X_{3}}{A_{2}}+\frac{X_{2}-X_{B}}{A_{3}}\right.\right. \\
& \left.\left.+\frac{A_{4}^{n+1}}{2 \bar{Y}_{2} A_{2}}+\frac{A_{G}^{n+1}}{2 \bar{Y}_{3} A_{3}}\right)\left(\frac{X_{2}-X_{3}}{C_{2-3}}\right)\right] \\
B_{j}= & A_{3}^{n+1}-\frac{1}{4} \frac{\Delta 1^{n+1 / 2}}{4 \pi \mu_{m}}\left[\left(\frac{Y_{3}-Y_{1}}{A_{2}}+\frac{Y_{4}-Y_{2}}{A_{2}}\right)\left(\frac{Y_{2}-Y_{3}}{C_{2-3}}\right)\right. \\
& +\left(\frac{Y_{1}-Y_{3}}{A_{4}}+\frac{Y_{2}-Y_{4}}{A_{1}}\right)\left(\frac{Y_{4}-Y_{1}}{C_{4-1}}\right) \\
& +\left(\frac{X_{3}-X_{1}}{A_{2}}+\frac{X_{4}-X_{2}}{A_{3}}+\frac{A_{G}^{n+1}}{2 \bar{Y}_{2} A_{2}}+\frac{A_{G}^{n+1}}{2 \bar{Y}_{3} A_{3}}\right)\left(\frac{X_{2}-X_{3}}{C_{2-3}}\right) \\
& \left.+\left(\frac{X_{1}-X_{3}}{A_{4}}+\frac{X_{2}-X_{4}}{A_{1}}+\frac{A_{(3)}^{n+1}}{2 \bar{Y}_{4} A_{4}}+\frac{A_{G}^{n+1}}{2 \bar{Y}_{1} A_{1}}\right)\left(\frac{X_{4}-X_{1}}{C_{4-1}}\right)\right]
\end{aligned}
$$




$$
\begin{aligned}
& C_{i}=-\frac{1}{4} \frac{\Delta t^{n+1 / 2}}{4 \pi \mu_{m}}\left[\left(\frac{Y_{11}-Y_{1}}{A_{4}}+\frac{Y_{4}-Y_{12}}{A_{1}}\right)\left(\frac{Y_{4}-Y_{1}}{C_{4-1}}\right)+\left(\frac{X_{11}-X_{1}}{A_{4}}\right.\right. \\
& \left.\left.+\frac{X_{4}-X_{32}}{A_{1}}+\frac{A_{0}^{n+1}}{2 \vec{Y}_{4} A_{4}}+\frac{A_{0}^{n+1}}{2 \bar{Y}_{1} A_{1}}\right)\left(\frac{X_{4}-X_{1}}{C_{4-1}}\right)\right] \\
& D_{i}=(H A)^{n}+\frac{1}{4} \frac{\Delta t^{n+1 / 2}}{4 \pi \mu_{m}}\left\{H _ { 0 } ^ { n } \left[\left(\frac{Y_{9}-Y_{4}}{A_{3}}\right)\left(\frac{Y_{2}-Y_{3}}{C_{2 \cdot 3}}\right)+\left(\frac{Y_{3}-Y_{10}}{A_{4}}\right)\left(\frac{Y_{4}-Y_{1}}{C_{4-1}}\right)\right.\right. \\
& \left.+\left(\frac{X_{9}-X_{4}}{A_{3}}+\frac{A_{0}^{n}}{2 \bar{Y}_{3} A_{3}}\right)\left(\frac{X_{2}-X_{3}}{C_{2-3}}\right)+\left(\frac{X_{3}-X_{10}}{A_{4}}+\frac{A_{0}^{n}}{2 \widetilde{Y}_{4} A_{4}}\right)\left(\frac{X_{4}-X_{1}}{C_{4-1}}\right)\right] \\
& +H_{\oplus}^{\mathrm{n}}\left[\left(\frac{Y_{1}-Y_{6}}{A_{2}}\right)\left(\frac{Y_{2}-Y_{3}}{C_{2-3}}\right)+\left(\frac{Y_{3}-Y_{2}}{A_{1}}\right)\left(\frac{Y_{4}-Y_{1}}{C_{4-1}}\right)\right. \\
& \left.+\left(\frac{X_{1}-X_{b}}{A_{2}}+\frac{A_{Q}^{n}}{2 \vec{Y}_{2} A_{2}}\right)\left(\frac{X_{2}-X_{3}}{C_{2-3}}\right)+\left(\frac{X_{5}-X_{2}}{A_{1}}+\frac{A_{0}^{n}}{2 \bar{Y}_{1} A_{1}}\right)\left(\frac{Y_{4}-X_{1}}{C_{4-1}}\right)\right] \\
& +H_{\Theta}^{n}\left[\left(\frac{Y_{12}-Y_{5}}{A_{1}}\right)\left(\frac{Y_{4}-Y_{1}}{C_{4-1}}\right)+\left(\frac{X_{12}-\lambda_{4}}{A_{1}}+\frac{A_{\Phi}^{n}}{2 \bar{Y}_{1} A_{1}}\right)\left(\frac{Y_{4}-Y_{1}}{C_{4-1}}\right)\right] \\
& +H_{\mathscr{D}}^{\mathrm{n}}\left[\left(\frac{Y_{6}-Y_{1}}{A_{2}}\right)\left(\frac{Y_{2}-Y_{3}}{C_{2-3}}\right)+\left(\frac{X_{6}-X_{7}}{A_{2}}+\frac{A_{0}^{n}}{2 Y_{2} A_{2}}\right)\left(\frac{X_{2}-X_{3}}{C_{2-3}}\right)\right] \\
& +H_{0}^{0}\left[\left(\frac{Y_{1}-Y_{9}}{A_{3}}\right)\left(\frac{Y_{2}-Y_{3}}{C_{2-3}}\right)+\left(\frac{X_{9}-X_{9}}{A_{3}}+\frac{A_{0}^{n}}{2 \bar{Y}_{3} A_{3}}\right)\left(\frac{X_{2}-X_{3}}{C_{2-3}}\right)\right] \\
& +1 H_{0}^{0}\left[\left(\frac{Y_{10}-Y_{11}}{A_{4}}\right)\left(\frac{Y_{4}-Y_{2}}{C_{4-1}}\right)+\left(\frac{X_{10}-X_{11}}{A_{4}}+\frac{A_{\Theta}^{\Omega}}{2 \bar{Y}_{4} A_{4}}\right)\left(\frac{X_{4}-X_{1}}{C_{4-1}}\right)\right] \text {. }
\end{aligned}
$$


AtPl:NDIX II

\section{THE:RMAL DIFFLISION ALCULATION}

\section{Cilculation Scheme for k-Sweep.}

First we must calculate $\nabla T$ to be used for the theinial flux change in the $k$-direction. Refer to Fig. B-1 for numbering of nodes and zones.

$$
\nabla T=\frac{\partial T}{\partial X} \vec{i}+\frac{\partial T}{\partial \bar{Y}} \vec{j}
$$

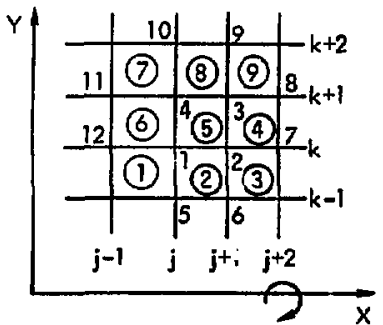

Fig. Ij-1. Calculational scheme for k-sweep.

a. $\left.\quad \frac{\partial T}{\partial Y}\right|_{1} ^{k}=\frac{+1}{2 A_{1}^{n+1 / 2}}\left[T_{\oplus}^{n+1}\left(X_{2}-X_{3}\right)+T_{\oplus}^{n}\left(X_{1}-X_{12}\right)+T_{\oplus}^{n}\left(X_{12}-X_{5}\right)+T_{\oplus}^{n+1}\left(X_{3}-X_{2}\right)\right]$

$$
\begin{aligned}
& \left.\frac{\partial T}{\partial X}\right|_{1} ^{k}=\frac{-1}{2:_{1}^{n+1 / 2}}\left[T_{G}^{n+1}\left(Y_{2}-Y_{4}\right)+T_{0}^{n}\left(Y_{4}-Y_{12}\right)+T_{0}^{n}\left(Y_{12}-Y_{5}\right)+T_{0}^{n+1}\left(Y_{5} \cdots Y_{2}\right)\right] \\
& R_{1}^{k}=\left.\frac{\partial T}{\partial X}\right|_{1} ^{k}: m_{1}^{k}=\left.\frac{\partial T}{\partial Y}\right|_{1} ^{k}
\end{aligned}
$$

b. $\left.\quad \frac{\partial T}{\partial Y}\right|_{2} ^{k}=\frac{+1}{2 A_{2}^{n+1 / 2}}\left[T_{Q}^{n}\left(X_{7}-X_{3}\right)+T_{G}^{n+1}\left(X_{3}-X_{1}\right)+T_{G}^{n+1}\left(X_{1}-X_{6}\right)+T_{G}^{n}\left(X_{6}-X_{7}\right)\right]$

$$
\left.\frac{\partial T}{\partial X}\right|_{2} ^{k}=\frac{-1}{2 A_{2}^{n+1 / 2}}\left[T_{0}^{n}\left(Y_{7}-Y_{3}\right)+T_{\oplus}^{n+1}\left(Y_{3}-Y_{1}\right)+T_{0}^{n+1}\left(Y_{1}-Y_{6}\right)+T_{G}^{n}\left(Y_{0}-Y_{7}\right)\right]
$$




$$
\begin{aligned}
& \ell_{2}^{k}=\left.\frac{\partial T}{\partial X}\right|_{2} ^{k} ; m_{2}^{k}=\left.\frac{\partial T}{\partial T}\right|_{2} ^{k} \\
& \text { c. }\left.\quad \frac{\partial T}{\partial Y}\right|_{3} ^{k}=\frac{+1}{2 A_{3}^{n+1 / 2}}\left[T_{\odot}^{n}\left(X_{B}-X_{9}\right)+T_{\oplus}^{n+1}\left(X_{9}-X_{4}\right)+T_{9}^{n+1}\left(X_{4}-X_{2}\right)+T_{9}^{n}\left(X_{2}-X_{4}\right)\right] \\
& \left.\frac{\partial T}{\partial X}\right|_{3} ^{k}=\frac{-1}{2 A_{3}^{n+1 / 2}}\left[T_{\mathscr{Q}}^{n}\left(Y_{B}-Y_{9}\right)+T_{\mathscr{Q}}^{n+1}\left(Y_{9}-Y_{4}\right)+T_{9}^{n+1}\left(Y_{4}-Y_{3}\right)+T_{\oplus}^{n}\left(Y_{2}-Y_{B}\right)\right] \\
& \mathbf{e}_{3}^{k}=\left.\frac{\partial T}{\partial \mathrm{X}}\right|_{3} ^{k}: \mathbf{m}_{3}^{k}=\left.\frac{\partial T}{\partial \mathrm{Y}}\right|_{3} ^{k} \\
& \text { d. }\left.\quad \frac{\partial T}{\partial Y}\right|_{4} ^{k}=\frac{+1}{2 A_{4}^{n+1 / 2}}\left[T_{\circledast 0}^{n+1}\left(X_{3}-X_{10}\right)+T_{0}^{n}\left(X_{10}-X_{11}\right)+T_{6}^{n}\left(X_{11}-X_{1}\right)+T_{6}^{n+1}\left(X_{1}-X_{3}\right)\right] \\
& \left.\frac{\partial T}{\partial X}\right|_{4} ^{k}=\frac{-1}{2 A_{4}^{n+1 / 2}}\left[T_{\oplus}^{n+1}\left(Y_{3}-Y_{10}\right)+T_{0}^{n}\left(Y_{10}-Y_{11}\right)+T_{0}^{n}\left(Y_{11}-Y_{1}\right)+T_{0}^{n+1}\left(Y_{1}-Y_{3}\right)\right] \\
& e_{4}^{k}=\left.\frac{3 T}{\partial X}\right|_{4} ^{k} ; m_{4}^{k}=\left.\frac{\partial T}{\partial Y}\right|_{4} ^{k}
\end{aligned}
$$

Having VT enables us to calculate the change in internal energy from fluxes in the k-direction (i.e. across 1.2 and $3-4$ in Fig. B-2).

$$
\begin{aligned}
& \mathbf{E}=\frac{\partial T}{\partial X} \\
& m=\frac{\partial T}{\partial Y} \\
& \Lambda=K+\left(\frac{4}{3} a_{R^{c}}\right) T^{3} \lambda
\end{aligned}
$$

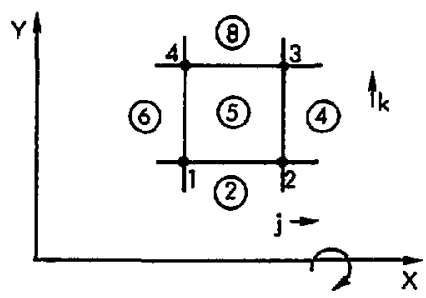

Fig. B-2. Calculational grid. 


$$
\begin{aligned}
& \text { i. }\left\{\begin{array}{l}
d T\left(\left.\frac{\partial E}{\partial T}\right|_{V}\right)=V \nabla \cdot(\Lambda \nabla T) d t+W \\
* W=\frac{1}{2}\left[\left(-P+q+\left.\frac{\partial E}{\partial V}\right|_{T}\right) d V+v \frac{J^{\prime}}{C} d t+d Z\right]
\end{array}\right. \\
& \text { b. }\left\{\begin{array}{l}
\nabla T=\ell \vec{i}+m \vec{j} \\
\nabla \cdot(\Lambda \nabla T)=\Lambda\left(\frac{\partial Q}{\partial X}+\frac{1}{Y} \frac{\partial Y m}{\partial Y}\right)
\end{array}\right. \\
& \text { c. } \int \Lambda \frac{\partial \ell}{\partial X}=-\frac{1}{2 A_{(3)}}\left[(\Lambda)_{12}\left(l_{1}^{k}+l_{2}^{k}\right)\left(Y_{1}-Y_{2}\right)+(\Lambda)_{3-4}\left(l_{3}^{k}+l_{4}^{k}\right)\left(Y_{3}-Y_{4}\right)\right] \\
& \frac{\Lambda_{1} \partial Y_{m}}{Y \partial Y}=\frac{1}{A_{G}\left(Y_{1}+Y_{2}+Y_{5}+Y_{4}\right)}\left[(\Lambda)_{2-2}\left(m_{1}^{k}+m_{2}^{k}\right)\left(X_{1}-X_{2}\right)\left(Y_{1}+Y_{2}\right)\right. \\
& \left.+(\Lambda)_{3-4}\left(m_{3}^{k}+m_{4}^{k}\right)\left(X_{3}-X_{4}\right)\left(Y_{3}+Y_{4}\right)\right]
\end{aligned}
$$

Rewriting Eq. (B-1):

$$
\begin{aligned}
& \text { a. }\left(T^{n+1}-T^{n}\right)_{G}\left(\left.\frac{\partial E}{\partial T}\right|_{V}\right)_{G}^{n} \\
& =\frac{\Delta t^{n+1 / 2}}{4 A_{G}^{n}+1 / 2} V_{G}^{n+1 / 2} \int T_{G}^{n+1}\left[\left(\frac{Y_{2}-Y_{4}}{A_{1}}+\frac{Y_{3}-Y_{1}}{A_{2}}\right)\left(Y_{1}-Y_{2}\right)(\Lambda)_{1-2}^{n}\right. \\
& +\left(\frac{Y_{4}-Y_{2}}{A_{3}}+\frac{Y_{1}-Y_{3}}{A_{4}}\right)\left(Y_{3}-Y_{4}\right)(\Lambda)_{3-4}^{n} \\
& +2\left(\frac{X_{2}-X_{4}}{A_{1}}+\frac{X_{3}-X_{1}}{A_{2}}\right)\left(X_{1}-X_{2}\right)\left(\frac{Y_{1}+Y_{2}}{Y_{1}+Y_{2}+Y_{3}+Y_{4}}\right)(\Lambda)_{1-2}^{n} \\
& \left.+2\left(\frac{X_{4}-X_{2}}{A_{3}}+\frac{X_{1}-X_{3}}{A_{4}}\right)\left(X_{3}-X_{4}\right)\left(\frac{X_{3}+X_{4}}{Y_{1}+Y_{2}+Y_{3}+Y_{4}}\right)(\Lambda)_{3-4}^{n}\right]
\end{aligned}
$$

*NOTE: The factor $1 / 2$ is because we are going to calculate the thermal diffusion energy change by adding the flux changes from two directions to abtain the total change; herce, the W/erms will appeat twice. 
$.9 \varepsilon^{-}$

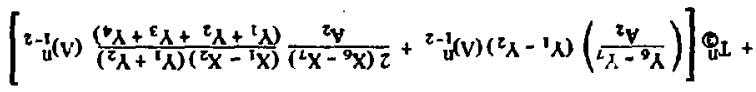

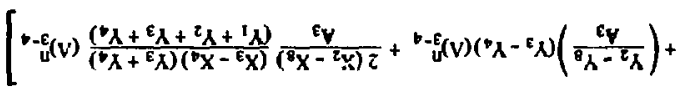

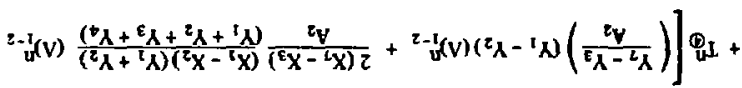

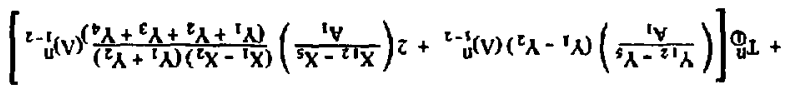

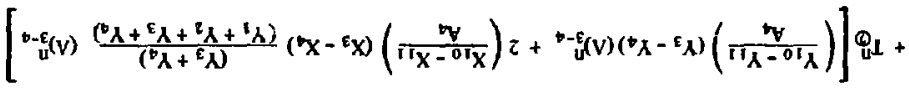

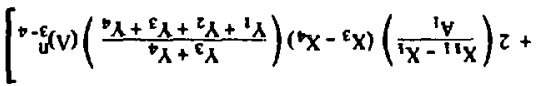

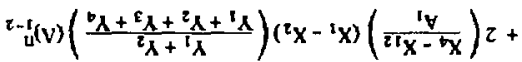

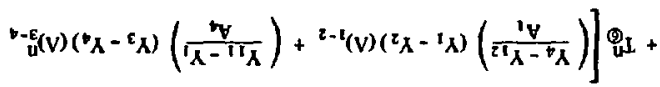

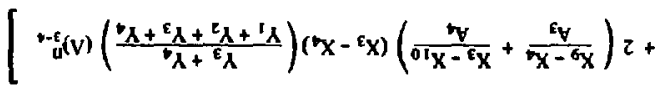

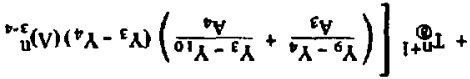

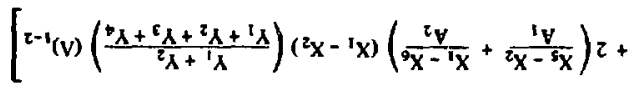

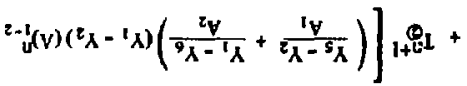




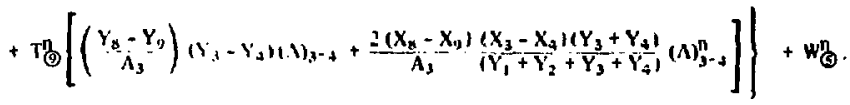

b. Where:

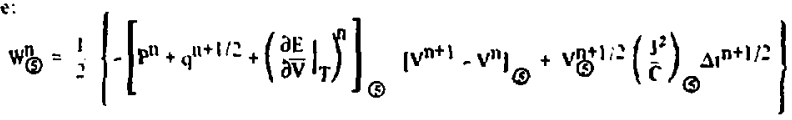

Sec Eq. (17) for $\mathrm{J}^{2} / \mathrm{C}$.

Referring to Fig, B.3 we see that for a given j-Jine:

$$
\begin{aligned}
& T_{i+1} \equiv T_{(0)}^{n+1}=T_{j+1 / 2, k+3 / 2}^{n+1} . \\
& T_{j} \equiv T_{(j)}^{n+1}=T_{j+1 / 2, k+1 / 2}^{n+1} . \\
& T_{i-1} \equiv T_{0}^{n+1}=T_{j+1 / 2, k-1 / 2}^{n+1} .
\end{aligned}
$$

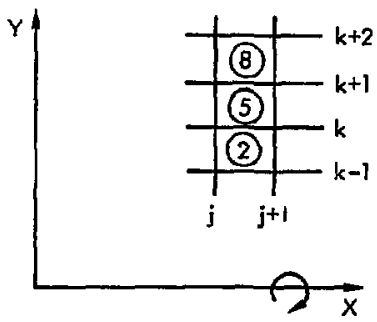

Fig. B-3. Grid for calculating temperature diffusion from contributions in the k-direction.

Rewriting again Eq. (B-2):

$$
A_{i} T_{i+1}+B_{i} T_{i}+C_{i} T_{i-1}=D_{i}
$$

where:

$$
\begin{aligned}
A_{j}= & \frac{-\Delta t^{n+l / 2}}{4 A_{(3)}^{n+1 / 2}} V_{G}^{n+1 / 2}\left[\left(\frac{Y_{9}-Y_{4}}{A_{3}}+\frac{Y_{3}-Y_{10}}{A_{4}}\right)\left(Y_{3}-Y_{4}\right)(\Lambda)_{3-4}^{n}\right. \\
& \left.+2\left(\frac{X_{9}-X_{4}}{A_{3}}+\frac{X_{3}-X_{10}}{A_{4}}\right) \frac{\left(X_{3}-X_{4}\right)\left(Y_{3}+Y_{4}\right)}{\left(Y_{1}+Y_{2}+Y_{3}+Y_{4}\right)}(\Lambda)_{3-4}^{n}\right]
\end{aligned}
$$




$$
\begin{aligned}
& B_{i}=\left(\left.\frac{\partial E}{\partial T}\right|_{V}\right)_{3}^{n}=\frac{\Delta t^{n+1 / 2}}{4 A_{G}^{n+1 / 2}} V_{G}^{n+1 / 2}\left[\left(\frac{Y_{2}-Y_{4}}{A_{1}}+\frac{Y_{3}-Y_{1}}{A_{2}}\right)\left(Y_{1}-Y_{2}\right)(\Lambda)_{1-2}^{n}\right. \\
& +\left(\frac{Y_{4}-Y_{3}}{A_{3}}+\frac{Y_{1}-Y_{3}}{A_{4}}\right)\left(Y_{3}-Y_{4}\right)(\Lambda)_{3-4}^{n} \\
& +2\left(\frac{X_{2}-X_{4}}{A_{1}}+\frac{X_{3}-X_{1}}{A_{2}}\right)\left(\frac{\left(X_{1}-X_{2}\right)\left(Y_{1}+Y_{2}\right)}{\left(Y_{1}+Y_{2}\right)+\left(Y_{3}+Y_{4}\right)}\right)(\Lambda)_{1-2}^{n} \\
& \left.+2\left(\frac{X_{4}-X_{2}}{A_{3}}+\frac{X_{1}-X_{3}}{A_{4}}\right)\left(\frac{\left(X_{3}-X_{4}\right)\left(Y_{3}+Y_{4}\right)}{Y_{1}+Y_{2}+Y_{3}+Y_{4}}\right)(\Lambda)_{3-4}^{n}\right] \text {, } \\
& c_{i}=\frac{-\Delta t^{n+1 / 2}}{4 A_{G}^{n+1 / 2}} V_{G}^{n+1 / 2}\left[\left(\frac{Y_{5}-Y_{2}}{A_{1}}+\frac{Y_{1}-Y_{6}}{A_{2}}\right)\left(Y_{1}-Y_{2}\right)(\Lambda)_{1-2}^{n}\right. \\
& \left.+2\left(\frac{X_{5}-X_{2}}{A_{1}}+\frac{X_{1}-X_{6}}{A_{2}}\right)\left(X_{1}-X_{2}\right)\left(\frac{Y_{1}+Y_{2}}{Y_{1}+Y_{2}+Y_{3}+Y_{4}}\right)(\Lambda)_{1-3}^{n}\right] \text {. } \\
& D_{i}=T_{G}^{n}\left(\left.\frac{\partial E}{\partial T}\right|_{V}\right)_{\Theta}^{n}+\frac{\Delta t^{n+1 / 2}}{4 A_{\Theta}^{n+1 / 2}} V_{\Theta}^{n+1 / 2}\left\{T _ { 0 } ^ { n } \left[\left(\frac{Y_{4}-Y_{12}}{A_{4}}\right)\left(Y_{1}-Y_{2}\right)(\Lambda)_{1-2}^{n}\right.\right. \\
& +\left(\frac{Y_{11}-Y_{1}}{A_{4}}\right)\left(Y_{3}-Y_{4}\right)(\Lambda)_{3-4}^{n} \\
& \left.+2\left(\frac{X_{4}-X_{12}}{A_{1}}\right) \frac{\left(X_{1}-X_{2}\right)\left(Y_{1}+Y_{2}\right)}{\left(Y_{1}+Y_{2}+Y_{3}+Y_{4}\right)}(\Lambda)_{1-2}^{n} \frac{+2\left(X_{11}-X_{3}\right)}{A_{4}} \frac{\left(X_{3}-X_{4}\right)\left(Y_{3}+Y_{4}\right)}{\left(Y_{1}+Y_{2}+Y_{3}+Y_{4}\right)}(\Lambda)_{3-4}^{n}\right] \\
& +T_{3}^{n}\left[\left(\frac{Y_{10}-Y_{11}}{A_{4}}\right)\left(Y_{3}-Y_{4}\right)(\Lambda)_{3-4}^{n}+2\left(\frac{X_{10}-X_{11}}{A_{4}}\right) \frac{\left(X_{3}-X_{4}\right)\left(Y_{3}+Y_{4}\right)}{\left(Y_{1}+Y_{2}+Y_{3}+Y_{4}\right)}(\Lambda)_{3-4}^{n}\right] \\
& +T^{n}\left[\left(\frac{Y_{12}-Y_{5}}{A_{1}}\right)\left(Y_{1}-Y_{2}\right)(\Lambda)_{1-2}^{n}+2\left(\frac{X_{12}-X_{5}}{A_{1}}\right) \frac{\left(X_{1}-X_{2}\right)\left(Y_{1}+Y_{2}\right)}{\left(Y_{1}+Y_{2}+Y_{3}+Y_{4}\right)}(\Lambda)_{1-2}^{n}\right] \\
& +T^{n}\left[\left(\frac{Y_{9}-Y_{3}}{A_{2}}\right)\left(Y_{1}-Y_{2}\right)(\Lambda)_{1-2}^{n}+2 \frac{\left(X_{7}-X_{3}\right)\left(X_{1}-X_{2}\right)\left(Y_{1}+Y_{2}\right)}{A_{2}\left(Y_{1}+Y_{2}+Y_{3}+Y_{4}\right)}(\Lambda)_{1-2}^{n}\right. \\
& \left.+\left(\frac{Y_{2}-Y_{8}}{A_{3}}\right)\left(Y_{3}-Y_{4}\right)(\Lambda)_{3-4}^{n !}+2 \frac{\left(X_{2}-X_{8}\right)\left(X_{3}-X_{4}\right)\left(Y_{3}+Y_{4}\right)}{A_{3}\left(Y_{1}+Y_{2}+Y_{3}+Y_{4}\right)}(\Lambda)_{3-4}^{n}\right]
\end{aligned}
$$


$+T_{(3)}^{\mathrm{n}}\left[\left(\frac{Y_{6}-Y_{7}}{A_{2}}\right)\left(Y_{1}-Y_{2}\right)(\Lambda)_{1-2}^{n}+\frac{2\left(X_{6}-X_{7}\right)\left(X_{1}-X_{2}\right)\left(Y_{1}+Y_{2}\right)}{A_{1}\left(Y_{1}+Y_{2}+Y_{3}+Y_{4}\right)}(\Lambda)_{1-2}^{n}\right]$
$\left.+T_{9}^{n}\left[\left(\frac{Y_{5}-Y_{9}}{A_{3}}\right)\left(Y_{3}-Y_{4}\right)(\Lambda)_{3-4}^{n}+2 \frac{\left(X_{8}-X_{9}\right)\left(X_{3}-X_{4}\right)\left(Y_{3}+Y_{4}\right)}{A_{3}\left(Y_{1}+Y_{2}+Y_{3}+Y_{4}\right)}(\Lambda)_{3-4}^{n}\right]\right\}$

$+w_{G}^{n}$,

and

$$
w_{G}^{n}=+\frac{1}{2}\left\{-\left[P^{n}+q^{n+1 / 2}+\left(\left.\frac{\partial E}{\partial V}\right|_{T}\right)^{n}\right]\left[v^{n+1}-v^{n}\right]+v_{G}^{n+1 / 2}\left(\frac{J^{2}}{c}\right)_{0}^{n} \Delta t^{n+1 / 2}+d Z\right\} \text {. }
$$

(See Ref. 1. for the calculation of dZ.)

To calculate Ohmic heating we expand $\mathrm{J}^{2} / \mathrm{C}$ as follows:

$$
\begin{aligned}
& \left(\frac{\mathrm{J}^{2}}{\mathrm{c}}\right)_{(5)}=\left[\frac{1}{2}\left(\frac{\mathrm{j}^{2}}{\mathrm{c}}\right)_{(5)}^{\mathrm{k}}+\left(\frac{\mathrm{J}^{2}}{\mathrm{c}}\right)_{(5)}^{\mathrm{j}}\right] \\
& \left(\frac{b^{2}}{C}\right)_{G}^{k}=\frac{c_{G}}{2 \cdot 16 \pi^{2}}\left[\left(\frac{a_{1}^{2}+a_{2}^{2}}{2}+\frac{b_{1}^{2}+b_{2}^{2}}{2}\right)\left(\frac{1}{c_{1 \cdot 2}}\right)^{2}\right. \\
& \left.+\left(\frac{a_{3}^{2}+a_{4}^{2}}{2}+\frac{b_{3}^{2}+b_{4}^{2}}{2}\right)\left(\frac{1}{c_{3-4}}\right)^{2}\right] \\
& \left(\frac{\mathrm{J}^{2}}{\mathrm{C}}\right)_{(5)}^{\mathrm{j}}=\frac{\mathrm{C}_{(5)}}{2 \cdot 16 \pi^{2}}\left[\left(\frac{\mathrm{a}_{2}^{2}+\mathrm{a}_{3}^{2}}{2}+\frac{b_{2}^{2}+\mathrm{b}_{3}^{2}}{2}\right)\left(\frac{1}{\mathrm{C}_{2-3}}\right)^{2}\right. \\
& \left.+\left(\frac{a_{4}^{2}+a_{1}^{2}}{2}+\frac{b_{4}^{2}+b_{1}^{2}}{2}\right)\left(\frac{1}{c_{4-1}}\right)^{2}\right]
\end{aligned}
$$

The superscripts $k$ and $j$ refer to the $k$ and $j$ sweeps, where:

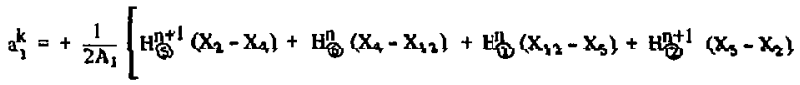

$$
\begin{aligned}
& \left.+\frac{(\mathrm{HA})_{5}^{\mathrm{n}+1}+(\mathrm{HA})_{0}^{\mathrm{O}}+(\mathrm{HA})_{0}^{\mathrm{H}}+(\mathrm{HA})_{0}^{\mathrm{R}}}{2 \mathrm{Y}_{1}}\right] \\
& a_{2}^{k}=+\frac{1}{2 A_{2}}\left[H_{6}^{n}\left(X_{7}-X_{3}\right)+H_{6}^{n+1}\left(X_{3}-X_{1}\right)+H_{6}^{n+1}\left(X_{1}-X_{6}\right)+H_{3}^{n}\left(X_{6}-X_{7}\right)\right. \\
& \left.+\frac{(H A)_{G}^{B}+(H A)_{O}^{n+1}+(H A)_{O}^{n+1}+(H A)_{O}^{B}}{2 \bar{Y}_{2}}\right]
\end{aligned}
$$




$$
\begin{aligned}
& a_{3}^{k}=+\frac{1}{2 A_{3}}\left[H_{3}^{n}\left(X_{8}-X_{9}\right)+H_{6}^{n+1}\left(X_{9}-X_{4}\right)+H_{0}^{n+1}\left(X_{4}-X_{2}\right)+H_{6}^{n}\left(X_{2}-X_{8}\right)\right. \\
& \left.+\frac{(H A)_{0}^{R}+(H A)_{0}^{n+1}+(H A)_{3}^{g+1}+(H A)^{n}}{2 \bar{Y}_{3}}\right)
\end{aligned}
$$$$
a_{4}^{k}=+\frac{1}{2 A_{4}}\left[H_{B}^{+1}\left(X_{3}-X_{10}\right)+H_{O}^{B}\left(X_{10}-X_{11}\right)+H_{G}^{n}\left(X_{11}-X_{1}\right)+H_{G}^{n+1}\left(X_{1}-X_{3}\right)\right.
$$$$
\left.+\frac{(H A)^{n+1}+(H A)^{M}+(H A)_{0}^{n}+(H A)^{n+1}}{2 \bar{Y}_{4}}\right]
$$

$$
\begin{aligned}
& b_{1}^{k}=\frac{-1}{2 A_{1}^{n+1 / 2}}\left[H_{S}^{n+1}\left(Y_{2}-Y_{4}\right)+H_{B}^{n}\left(Y_{4}-Y_{12}\right)+H_{O}^{B}\left(Y_{12}-Y_{5}\right)+H_{S}^{n+1}\left(Y_{5}-Y_{2}\right)\right] \\
& b_{2}^{k}=\frac{-1}{2 A_{1}^{n+1 / 2}}\left[H_{G}^{n}\left(Y_{7}-Y_{3}\right)+H_{G}^{n+1}\left(Y_{3}-Y_{1}\right)+H_{3}^{n+1}\left(Y_{1}-Y_{6}\right)+H_{G}^{n}\left(Y_{6}-Y_{7} i\right]\right. \\
& b_{3}^{k}=\frac{-1}{2 A_{3}^{n+1 / 2}}\left[H_{Q}^{n}\left(Y_{8}-Y_{9}\right)+H_{G}^{n+1}\left(Y_{9}-Y_{4}\right)+H_{G}^{n+1}\left(Y_{4}-Y_{2}\right)+H_{G}^{n}\left(Y_{2}-Y_{B}\right)\right] \\
& b_{4}^{k}=\frac{-1}{2 A^{n+1 / 2}}\left[H^{n+1}\left(Y_{3}-Y_{10}\right)+H_{O}^{B}\left(Y_{10}-Y_{11}\right)+H^{n}\left(Y_{11}-Y_{1}\right)+H^{n+1}\left(Y_{1}-Y_{3}\right)\right]
\end{aligned}
$$

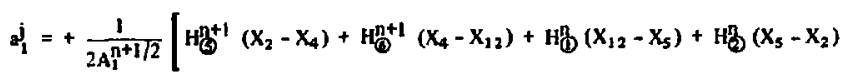

$$
\begin{aligned}
& \left.+\frac{\left.(H A)_{B}^{n+1}+(H A)_{0}^{n+1}+(H A)_{D}^{B}+(H A)_{0}^{n}\right)}{2 \bar{Y}_{1}}\right] \\
& \omega_{2}^{j}=+\frac{1}{2 A_{2}^{n+1 / 2}}\left[H_{\theta}^{n+1}\left(X_{7}-X_{3}\right)+H_{G}^{n+1}\left(x_{3}-X_{1}\right)+H_{3}^{n}\left(X_{1}-X_{6}\right)+H_{3}^{n}\left(X_{6}-X_{7}\right)\right. \\
& \left.\frac{(H A)_{\mathcal{G}}^{n+1}+(H A)_{\mathcal{S}}^{n+1}+(H A)_{(B)}^{n}+(H A)_{\mathcal{B}}^{n}}{2 \bar{Y}_{2}}\right]
\end{aligned}
$$




$$
\begin{aligned}
& y_{3}^{j}=+\frac{1}{2 A_{3}^{n+1 / 2}}\left[H_{9}^{n}\left(X_{8}-X_{9}\right)+H_{9}^{n}\left(X_{9}-X_{4}\right)+H_{9}^{n+1}\left(X_{4}-X_{2}\right)+H_{9}^{n+1}\left(X_{2}-X_{B}\right)\right. \\
& \left.+\frac{(H A)_{9}^{M}+(H A)_{(B)}^{n}+(H A)_{(9)}^{n+1}+(H A)_{(9)}^{n+1}}{2 \bar{Y}_{3}}\right] \\
& a_{4}^{j}=+\frac{1}{2 A_{4}^{n+1 / 2}}\left[H_{\theta}^{n}\left(X_{3}-X_{10}\right)+H_{O}^{\prime \prime}\left(X_{10}-X_{11}\right)+H_{3}^{n+1}\left(X_{11}-X_{1}\right)+H_{G}^{n+1}\left(X_{1}-X_{3}\right)\right. \\
& \left.+\frac{(H A)_{B}^{n}+(H A)_{O}^{n}+(H A)_{B}^{n+1}+(H A)_{S}^{n+1}}{2 \bar{Y}_{4}}\right] \\
& b_{1}^{j}=-\frac{1}{2 A_{1}^{n+1 / 2}}\left[H_{G}^{n+1}\left(Y_{2}-Y_{4}\right)+H_{\Theta}^{n+1}\left(Y_{4}-Y_{12}\right)+H_{\Theta}^{n}\left(Y_{12}-Y_{5}\right)+H_{C}^{n}\left(Y_{5}-Y_{2}\right)\right] \\
& b_{2}^{j}=-\frac{1}{2 A_{2}^{n+1 / 2}}\left[H_{\odot}^{n+1}\left(Y_{7}-Y_{3}\right)+H_{\odot}^{n+1}\left(Y_{3}-Y_{1}\right)+H_{\Theta}^{n}\left(Y_{1}-Y_{6}\right)+H_{\odot}^{n}\left(Y_{6}-Y_{7}\right)\right] \\
& b_{3}^{j}=-\frac{1}{2 A_{3}^{n+1 / 2}}\left[H_{9}^{n}\left(Y_{8}-Y_{9}\right)+H_{9}^{n}\left(Y_{9}-Y_{4}\right)+H_{\odot}^{n+1}\left(Y_{4}-Y_{2}\right)+H_{9}^{n+1}\left(Y_{2}-Y_{8}\right)\right]
\end{aligned}
$$

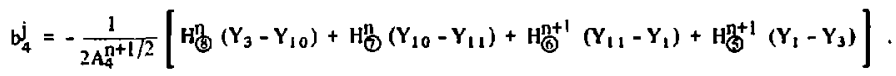

\section{Calculation Sclieme for $\mathrm{j}$-Sweep.}

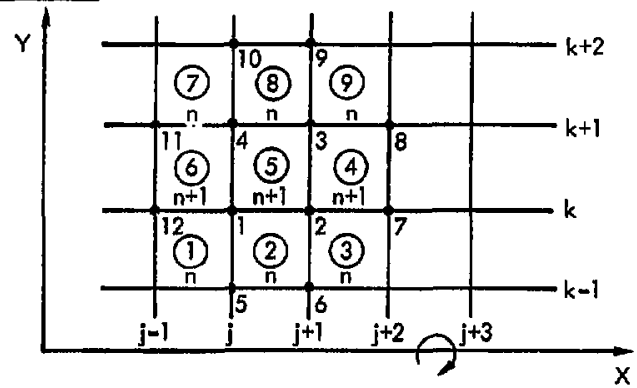

Fig. B-4. Calculational grid for $\mathrm{j}$-sweep. 
Calculation of $\nabla T$ to be used for the thermal flux change in the j-direction proceeds as follows. See Fig. B-4 for time and position number scheme.

$$
\nabla T=\frac{\partial T}{\partial X} \vec{i}+\frac{\partial T}{\partial Y} \vec{j}
$$

a. $\left.\quad \frac{\partial T}{\partial \bar{Y}}\right|_{1} ^{j}=+\frac{1}{2 A_{1}^{n+1 / 2}}\left[T_{\mathscr{G}}^{+1}\left(X_{2}-X_{4}\right)+T_{\mathscr{O}}^{n+1}\left(X_{4}-X_{12}\right)+T_{D}^{n}\left(X_{12}-X_{5}\right)+T_{\mathscr{Q}}^{n}\left(X_{5}-X_{2}\right)\right]$

$$
\begin{aligned}
& \left.\frac{\partial T}{\partial X}\right|_{1} ^{j}=\frac{-1}{2 A_{1}^{n+1 / 2}}\left[T_{S}^{n+1}\left(Y_{2}-Y_{4}\right)+T_{0}^{n+1}\left(Y_{4}-Y_{12}\right)+T_{D}\left(Y_{12}-Y_{5}\right)+T_{6}^{n}\left(Y_{5}-Y_{2}\right)\right] \\
& \mathrm{R}_{1}^{j}=\left.\frac{\partial T}{\partial X}\right|_{1} ^{j}: m_{1}^{j}=\left.\frac{\partial T}{\partial Y}\right|_{1} ^{j}
\end{aligned}
$$

b. $\left.\left.\quad \frac{\partial T}{\partial Y}\right|_{1} ^{j}=\frac{+1}{2 A_{2}^{n+1 / 2}} \mid T_{S}^{n+1}\left(X_{7}-X_{3}\right)+T_{G}^{n+1}\left(X_{3}-X_{1}\right) T_{G}^{n}\left(X_{1}-X_{6}\right)+T_{G}^{n}\left(X_{6}-X_{7}\right)\right]$

$$
\begin{aligned}
& \left.\frac{\partial T}{\partial X}\right|_{2} ^{j}=-\frac{1}{2 A_{2}^{n+1 / 2}}\left[T_{6}^{n^{+1}}\left(Y_{7}-Y_{3}\right)+T_{6}^{n+1}\left(Y_{3}-Y_{1}\right)+T_{3}^{\eta}\left(Y_{1}-Y_{6}\right)+T_{6}^{n}\left(Y_{6}-Y_{7}\right)\right] \\
& Q_{2}^{j}=\left.\frac{\partial T}{\partial X}\right|_{2} ^{j}: m_{2}^{j}=\left.\frac{\partial T}{\partial \bar{Y}}\right|_{2} ^{j}
\end{aligned}
$$

c. $\left.\frac{\partial T}{\partial Y}\right|_{3} ^{j}=\frac{+1}{2 A_{3}^{n+1} T / 2}\left[T_{G}^{g}\left(X_{8}-X_{9}\right)+T_{G}^{\eta}\left(X_{9}-X_{4}\right)+T_{G}^{+1}\left(X_{4}-X_{2}\right)+T_{G}^{n+1}\left(X_{2}-X_{B}\right)\right]$

$$
\begin{aligned}
& \left.\frac{\partial T}{\partial X}\right|_{3} ^{j}=\frac{-1}{2 A_{3}^{n+1 / 2}}\left[T_{b}\left(Y_{8}-Y_{9}\right)+T_{6}\left(Y_{9}-Y_{4}\right)+T_{3}^{n+1}\left(Y_{4}-Y_{2}\right)+T^{n+1}\left(Y_{2}-Y_{8}\right)\right] \\
& e_{3}^{j}=\left.\frac{\partial T}{\partial X}\right|_{3} ^{j}: m_{3}^{j}=\left.\frac{\partial T}{\partial Y}\right|_{3} ^{j}
\end{aligned}
$$


J. $\left.\quad \frac{\partial T}{\partial Y}\right|_{4} ^{j}=+\frac{1}{2 A_{4}^{n+1 / 2}}\left[T_{(0)}^{n}\left(X_{3}-X_{10}\right)+T_{O}^{n}\left(X_{10}-X_{11}\right)+T_{0}^{n+1}\left(X_{11}-X_{1}\right)+T_{\mathscr{O}}^{n+1}\left(X_{1}-X_{3}\right)\right]$

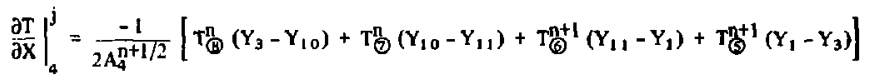

$$
\begin{aligned}
& \ell_{4}^{j}=\left.\frac{\partial T}{\partial X}\right|_{4} ^{j} ; m_{4}^{j}=\left.\frac{\partial T}{\partial Y}\right|_{4} ^{j}
\end{aligned}
$$

With $\nabla T$ from the above wa can calculate the change in internal energy from fluxes in the $j$-direction (i.e. across 2-3 and 4-1 in Fig. B-5).

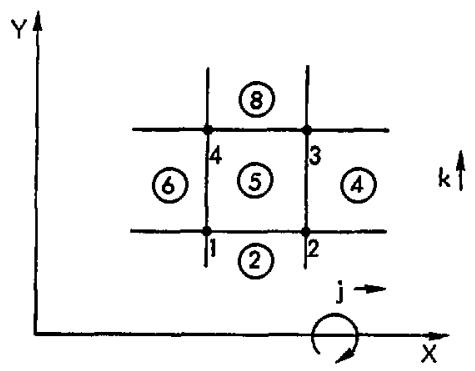

Fig. B.S. Calculational grid.

$$
\begin{gathered}
d T\left(\left.\frac{\partial E}{\partial T}\right|_{V}\right)=V \nabla \cdot(\Lambda \nabla T) d t+W \\
* W=\frac{1}{2}\left[-\left(P+q+\left.\frac{\partial E}{\partial V}\right|_{T}\right) d V+V \frac{J^{2}}{C} d t+d z\right]
\end{gathered}
$$

But

$$
\nabla T=\ell \vec{i}+m \vec{j}
$$

and

*NOTE: The factor $(1 / 2)$ is present because the total energy change by thermal diffusion will be calculated by adding the flux changes from two directions. Hence the $W$ terms will appear twice. 


$$
\nabla \cdot(\Lambda \nabla T)=\Lambda\left(\frac{\partial \ell}{\partial X}+\frac{1}{Y} \frac{\partial Y m}{\partial Y}\right)
$$

where

$$
\begin{aligned}
& \ell=\frac{\partial T}{\partial \bar{X}} \\
& \mathrm{~m}=\frac{\partial T}{\partial Y} \\
& \Lambda=K+\left(\frac{4}{3} \mathrm{a}^{\mathrm{a}}\right) \mathbf{T}^{3} \lambda .
\end{aligned}
$$

Therefore

$$
\Lambda \frac{\partial \ell}{\partial X}=-\frac{1}{\partial A_{G}}\left[(\Lambda)_{2-3}^{n}\left(Q l_{2}^{j}+Q \dot{j}_{3}\right)\left(Y_{2}-Y_{3}\right)+(\Lambda)_{4-1}^{n}\left(Q \frac{j_{4}}{2}+Q j_{1}\right)\left(Y_{4}-Y_{1}\right)\right]
$$

and

$$
\begin{aligned}
\wedge \frac{1}{Y} \frac{\partial Y_{m}}{\partial \bar{Y}}= & \frac{1}{A_{(G)}\left(Y_{1}+Y_{2}+Y_{3}+Y_{4}\right)}\left[(\Lambda)_{2-3}^{n}\left(m \frac{j}{j}+m_{3}^{j}\right)\left(X_{2}-X_{3}\right)\left(Y_{2}+Y_{3}\right)\right. \\
& \left.+(\Lambda)_{4-1}^{n}\left(m_{4}^{j}+m_{1}^{j}\right)\left(X_{4}-X_{1}\right)\left(Y_{4}+Y_{1}\right)\right] .
\end{aligned}
$$

Rewriting Eq. (B-4)

$$
\begin{aligned}
& \left(T^{n+1}-T^{n}\right)_{G}\left(\left.\frac{\partial E}{\partial T}\right|_{V}\right)_{O}^{n} \\
& =\frac{\Delta 1^{n+1 / 2}}{4 A_{G}^{n+1 / 2}} V_{G}^{n+1 / 2}\left\{T _ { G } ^ { n + 1 } \left[\left(\frac{Y_{1}-Y_{1}}{A_{2}}+\frac{Y_{4}-Y_{2}}{A_{3}}\right)\left(Y_{2}-Y_{3}\right)(\Lambda)_{2-3}^{n}\right.\right. \\
& +\left(\frac{Y_{1}-Y_{3}}{A_{4}}+\frac{Y_{2}-Y_{4}}{A_{1}}\right)\left(Y_{4}-Y_{1}\right)(\Lambda)_{4-1}^{n} \\
& \quad \cdot \quad 2\left(\frac{X_{3}-X_{1}}{A_{2}}+\frac{X_{4}-X_{2}}{A_{3}}\right)\left(X_{2}-X_{3}\right)\left(\frac{Y_{2}+Y_{3}}{Y_{1}+Y_{2}+Y_{3}+Y_{4}}\right)(\Lambda)_{2-3}^{n} \\
& \quad+2\left(\frac{X_{1}-X_{3}}{A_{4}}+\frac{X_{2}-X_{4}}{A_{1}}\right)\left(X_{4}-X_{1}\right)\left(\frac{Y_{4}-Y_{1}}{Y_{1}+Y_{1}+Y_{3}+Y_{4}}\right)(\Lambda)_{4-1}^{n !}
\end{aligned}
$$

44. 


$$
\begin{aligned}
& +T_{9}^{\pi+1}\left[\left(\frac{Y_{1}-Y_{3}}{A_{2}}+\frac{Y_{2}-Y_{B}}{A_{3}}\right)\left(Y_{2}-Y_{3}\right)(\Lambda)_{2-3}^{n}\right. \\
& \left.+2\left(\frac{X_{7}-X_{3}}{A_{2}}+\frac{X_{3}-X_{8}}{A_{3}}\right)\left(X_{2}-X_{3}\right)\left(\frac{Y_{2}+Y_{3}}{Y_{1}+Y_{2}+Y_{3}+Y_{4}}\right)(\Lambda)_{2-3}^{n}\right] \\
& +T_{6}^{n+1}\left\{\left(\frac{Y_{11}-Y_{1}}{A_{4}}+\frac{Y_{4}-Y_{12}}{A_{1}}\right)\left(Y_{4}-Y_{1}\right)(\Lambda)_{4-1}^{n}\right. \\
& +2\left(\frac{X_{11}-X_{1}}{A_{4}}+\frac{X_{4}-X_{12}}{A_{1}}\right)\left(X_{4}-X_{1}\right)\left(\frac{Y_{4}+Y_{1}}{Y_{1}+Y_{2}+Y_{3}+Y_{4}}\right)(\Lambda)_{4-1}^{n} \\
& +T_{0}^{n}\left[\left(\frac{Y_{1}-Y_{6}}{A_{2}}\right)\left(Y_{2}-Y_{3}\right)(\Lambda)_{2-3}^{n}+\left(\frac{Y_{5}-Y_{2}}{A_{1}}\right)\left(Y_{4}-Y_{1}\right)(\Lambda)_{4-1}^{n}\right. \\
& +2\left(\frac{X_{1}-X_{6}}{A_{2}}\right)\left(X_{2}-X_{3}\right)\left(\frac{Y_{2}+Y_{3}}{Y_{1}+Y_{2}+Y_{3}+Y_{4}}\right)(\Lambda)_{2-3}^{n} \\
& \left.+2\left(\frac{X_{5}-X_{2}}{A_{1}}\right)\left(X_{4}-X_{1}\right)\left(\frac{Y_{4}+Y_{4}}{Y_{1}+Y_{2}+Y_{3}+Y_{4}}\right)(\Lambda)_{4-2}^{n}\right] \\
& +T^{n}\left[\left(\frac{Y_{9}-Y_{4}}{A_{3}}\right)\left(Y_{2}-Y_{3}\right)(\Lambda)_{2-3}^{n}+\left(\frac{Y_{3}-Y_{10}}{A_{4}}\right)\left(N_{4}-Y_{1}\right)(\Lambda)_{4-3}^{n}\right. \\
& \left.+2\left(\frac{X_{9}-X_{4}}{A_{3}}\right)\left(X_{2}-X_{3}\right)\left(\frac{Y_{2}+Y_{3}}{Y_{1}+Y_{2}+Y_{3}+Y_{4}}\right)(\Lambda)_{2-3}^{n}+2\left(\frac{X_{3}-X_{10}}{A_{4}}\right)\left(X_{4}-X_{1}\right)\left(\frac{Y_{4}+Y_{3}}{Y_{1}+Y_{2}+Y_{3}+Y_{4}}\right)(\Lambda)_{4-1}^{n}\right) \\
& +T^{n}\left[\left(\frac{Y_{12}-Y_{5}}{A_{1}}\right)\left(Y_{4}-Y_{1}\right)(\Lambda)_{4-1}^{n}+2\left(\frac{X_{12}-X_{5}}{A_{1}}\right)\left(X_{4}-\check{X}_{1}\right)\left(\frac{Y_{4}+Y_{1}}{Y_{1}+Y_{2}+Y_{3}+Y_{4}}\right)(\Lambda)_{4-1}^{n}\right] \\
& +T_{3}^{n}\left[\left(\frac{Y_{6}-Y_{7}}{A_{2}}\right)\left(Y_{2}-Y_{3}\right)(\Lambda)_{2-3}^{n}+2\left(\frac{X_{5}-X_{2}}{A_{2}}\right)\left(X_{2}-X_{3}\right)\left(\frac{Y_{2}+Y_{3}}{Y_{1}+Y_{2}+Y_{3}+Y_{A}}\right)(\Lambda)_{2-3}^{n}\right] \\
& +T^{3}\left[\left(\frac{Y_{3}-Y_{9}}{A_{3}}\right)\left(Y_{2}-Y_{3}\right)(\Lambda)_{2-3}^{n}+2\left(\frac{X_{B}-X_{9}}{A_{3}}\right)\left(X_{2}-X_{3}\right)\left(\frac{Y_{2}+Y_{3}}{Y_{1}+Y_{2}+Y_{3}+Y_{4}}\right)(\Lambda)_{2-3}^{n}\right]
\end{aligned}
$$

45. 


$$
\left.+T_{3}\left[\left(\frac{Y_{10}-Y_{21}}{A_{4}}\right)\left(Y_{4}-Y_{1}\right)(\Lambda)_{4-1}^{n}+2\left(\frac{X_{10}-X_{11}}{A_{4}}\right)\left(X_{4}-X_{1}\right)\left(\frac{Y_{4}+Y_{1}}{Y_{1}+Y_{2}+Y_{3}+Y_{4}}\right)(\Lambda)_{4-1}^{n}\right]\right\}
$$

+ why

(B-5)

where:

$$
\begin{aligned}
w^{2} & =\frac{l}{2}\left\{-\left[P^{n} \div q^{n+1 / 2}+\left(\frac{\partial E}{\partial V} L_{T}\right)^{n}\right]_{\Theta}\left(v^{n+1}-v^{n}\right)_{\odot}\right. \\
& \left.+v_{S}^{n+1 / 2}\left(\frac{3^{2}}{c}\right)_{\odot}^{n} \Delta 1^{n+1 / 2}\right\}
\end{aligned}
$$

See Eq. (B-3) for $]^{3} / C$.

For a given kline, as shown in Fig. B.-6:

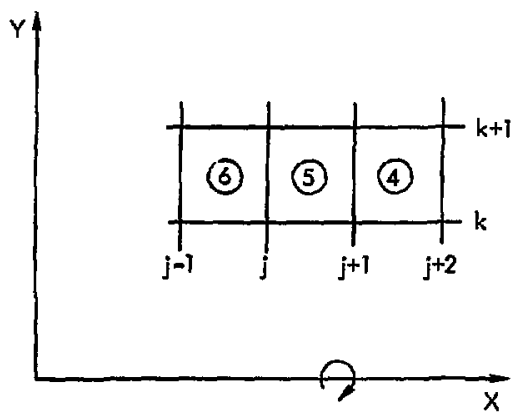

Fig. B-6. Grid for calculating temperature flux changes in zone @ from contributions in the j-direction.

$$
\begin{aligned}
& T_{i+1} \equiv T_{\oplus}^{n+1}=T_{j+3 / 2, k+1 / 2}^{n+1} \\
& T_{i} \equiv T_{G}^{n+1}=T_{j+1 / 2, k+1 / 2}^{n+1} \\
& T_{i-1} \equiv T_{\oplus}^{+1}=T_{j-1}^{n+1 / 2, k+1 / 2}
\end{aligned}
$$

Rewriting again Eq. (B.5):

$$
A_{i} T_{i+1}+B_{i} T_{i}+C_{i} T_{i-1}=D_{i},
$$

$-46$. 
where

$$
\begin{aligned}
A_{i}= & -\frac{\Delta t^{n+1 / 2}}{4 A_{G}^{n+1 / 2}} v_{G}^{n+1 / 2}\left[\left(\frac{Y_{7}-Y_{3}}{A_{2}}+\frac{Y_{2}-Y_{8}}{A_{3}}\right)\left(Y_{2}-Y_{3}\right)(\Lambda)_{2-3}^{n}\right. \\
& \left.+2\left(\frac{X_{7}-X_{3}}{A_{2}}+\frac{X_{2}-X_{8}}{A_{3}}\right)\left(X_{2}-X_{3}\right)\left(\frac{Y_{2}+Y_{3}}{Y_{1}+Y_{2}+Y_{3}+Y_{4}}\right)(\Lambda)_{2-3}^{n}\right] \\
B_{i}= & \left(\left.\frac{\partial E}{\partial T}\right|_{V} ^{n}-\frac{\Delta t^{n+1 / 2}}{4 A_{3}^{n+1 / 2}} V_{S}^{n+1 / 2}\left[\left(\frac{Y_{3}-Y_{1}}{A_{2}}+\frac{Y_{4}-Y_{2}}{A_{3}}\right)\left(Y_{2}-Y_{3}\right)(\Lambda)_{2-3}^{n}\right.\right. \\
& +\left(\frac{Y_{1}-Y_{3}}{A_{4}}+\frac{Y_{2}-Y_{4}}{A_{1}}\right)\left(Y_{4}-Y_{1}\right)(\Lambda)_{4-1}^{n} \\
& +2\left(\frac{X_{3}-X_{1}}{A_{2}}+\frac{X_{4}-X_{2}}{A_{3}}\right)\left(X_{2}-X_{3}\right)\left(\frac{Y_{2}+Y_{3}}{Y_{1}+Y_{2}+Y_{3}+Y_{4}}\right)(\Lambda)_{2-3}^{n} \\
& \left.+2\left(\frac{X_{1}-X_{3}}{A_{4}}+\frac{X_{2}-X_{4}}{A_{1}}\right)\left(X_{4}-X_{1}\right)\left(\frac{Y_{4}+Y_{1}}{Y_{1}+Y_{2}+Y_{3}+Y_{4}}\right)(\Lambda)_{4-1}^{n}\right] \\
& \left.+2\left(\frac{X_{11}-X_{1}}{A_{4}}+\frac{X_{4}-X_{12}}{A_{1}}\right)\left(X_{4}-X_{1}\right)\left(\frac{Y_{4}+Y_{1}}{Y_{1}+Y_{2}+Y_{3}+Y_{4}}\right)(\Lambda)_{4-1}^{n}\right] \\
C_{i}= & -\frac{\Delta t^{n+1 / 2}}{4 A_{G}^{n+1} / 2} V_{S}^{n+1 / 2}\left[\left(\frac{Y_{11}-Y_{1}}{A_{4}}+\frac{Y_{4}-Y_{12}}{A_{1}}\right)\left(Y_{4}-Y_{1}\right)(\Lambda)_{4-1}^{n}\right. \\
&
\end{aligned}
$$

and

$$
\begin{aligned}
D_{1}= & T_{G}^{n}\left(\left.\frac{\partial E}{\partial T}\right|_{V}\right)_{G}^{n}+\frac{\Delta 1^{n+1 / 2}}{4 A_{(3)}^{n+1 / 2}} V_{G}^{n+1 / 2} \mid T_{6}^{n}\left[\left(\frac{Y_{1}-Y_{6}}{A_{2}}\right)\left(Y_{2}-Y_{3}\right)(\Lambda)_{2-3}^{n}\right. \\
& +\left(\frac{Y_{5}-Y_{2}}{A_{1}}\right)\left(Y_{4}-Y_{1}\right)(\Lambda)_{4-1}^{n} \\
& +2\left(\frac{X_{1}-X_{6}}{A_{2}}\right)\left(X_{2}-X_{3}\right)\left(\frac{Y_{2}+Y_{3}}{Y_{1}+Y_{2}+Y_{3}+Y_{4}}\right)(\Lambda)_{2-3}^{n}
\end{aligned}
$$

47. 


$$
\begin{aligned}
& \left.+2\left(\frac{X_{5}-X_{2}}{A_{1}}\right)\left(X_{4}-X_{1}\right)\left(\frac{Y_{4}+Y_{2}}{Y_{1}+Y_{2}+Y_{3}+Y_{4}}\right)(\Lambda)_{4-1}^{n}\right] \\
& +T^{n}\left[\left(\frac{Y_{9}-Y_{4}}{A_{3}}\right)\left(Y_{2}-Y_{3}\right)(\Lambda)_{2-3}^{n}+\left(\frac{Y_{3}-Y_{10}}{A_{4}}\right)\left(Y_{4}-Y_{1}\right)(\Lambda)_{4-1}^{n}\right. \\
& +2\left(\frac{X_{9}-Y_{4}}{A_{3}}\right)\left(X_{2}-X_{3}\right)\left(\frac{Y_{2}+Y_{3}}{Y_{1}+Y_{2}+Y_{3}+Y_{4}}\right)\left(Y_{2-3}^{n}+2\left(\frac{X_{3}-Y_{10}}{A_{4}}\right)\left(X_{4}-X_{1}\right)\left(\frac{Y_{4}+Y_{1}}{Y_{1}+Y_{2}+Y_{3}+Y_{4}}\right)(\Lambda)_{4-1}^{n}\right] \\
& +T_{0}^{n}\left[\left(\frac{Y_{12}-Y_{5}}{A_{1}}\right)\left(Y_{4}-Y_{1}\right)(\Lambda)_{4-1}^{n}+2\left(\frac{X_{12}-X_{5}}{A_{1}}\right)\left(X_{4}-X_{1}\right)\left(\frac{Y_{4}+Y_{1}}{Y_{1}+Y_{2}+Y_{3}+Y_{4}}\right)(\Lambda)_{4-1}^{n}\right] \\
& +T_{0}^{n}\left[\left(\frac{Y_{6}-Y_{4}}{A_{2}}\right)\left(Y_{2}-Y_{3}\right)(\Lambda)_{2-3}^{n}+2\left(\frac{X_{6}-X_{7}}{A_{2}}\right)\left(X_{2}-X_{3}\right)\left(\frac{Y_{2}+Y_{3}}{Y_{1}+Y_{2}+Y_{3}+Y_{4}}\right)(\Lambda)_{2-3}^{n}\right] \\
& +T_{9}^{n}\left[\left(\frac{Y_{3}-Y_{9}}{A_{3}}\right)\left(Y_{2}-Y_{3}\right)(\Lambda)_{2-3}^{n}+2\left(\frac{X_{8}-X_{9}}{A_{3}}\right)\left(X_{2}-X_{3}\right)\left(\frac{Y_{2}+Y_{3}}{Y_{1}+Y_{2}+Y_{3}+Y_{4}}\right)(\Lambda)_{2-3}^{n}\right] \\
& \left.+T_{3}^{n}\left[\left(\frac{Y_{10}-Y_{11}}{A_{4}}\right)\left(Y_{4}-Y_{2}\right)(\Lambda)_{4-1}^{n}+2\left(\frac{X_{10}-X_{11}}{A_{4}}\right)\left(X_{4}-X_{1}\right)\left(\frac{Y_{4}+Y_{1}}{Y_{1}+Y_{2}+Y_{3}+Y_{4}}\right)(\Lambda)_{4-1}^{n}\right]\right]
\end{aligned}
$$

+ W 


\section{APPEND]X C \\ BACKWARD SUBSTITUTION METHOD FOR SOLVING A SYSTEM \\ OF LINEAR EQUATIONS OF THE FORN: $A_{i} H_{i+1}+B_{i} H_{i}+C_{i} H_{1-1}=D_{i}$}

Given:

$$
A_{i} H_{i+1}+B_{i} H_{i}+C_{i} H_{i-1}=D_{i} \text {; }
$$

$H_{i \max }$ and $H_{i \text { min }}$ are known quantities.

Define:

$$
E_{j+1} H_{i}+F_{i+1}=H_{i+1} \text {. }
$$

Substitute (C-2) into (C-1):

$$
A_{i}\left(E_{i+1} H_{i}+F_{i+1}\right)+B_{i} H_{i}+C_{i} H_{i-1}=D_{i} \text {, }
$$

or

$$
H_{j}=\frac{D_{i}-A_{i} F_{i+1}-C_{i} H_{i-1}}{A_{i} E_{i+1}+B_{i}}
$$

Rewrite Eq. (C-2) as

$$
H_{i}=E_{i} H_{i-1}+F_{i} \text {. }
$$

Equate coefficients of (C-3) and (C-4):

$$
E_{i}=\frac{-C_{i}}{A_{i} E_{i+1}+B_{i}} ; \quad F_{i}=\frac{D_{i}-A_{i} F_{i+1}}{A_{i} E_{i+1}+B_{i}}
$$

for:

$$
i=i_{\max -1} \text { to } j=i_{\min +1}
$$

where

$$
E_{i \max }=0 \text { and } F_{i \max }=H_{1 \max } .
$$

Store $E_{j}$ and $F_{i}$, then calculate $H_{i}$ for $i=i_{\min +1}$ to $i=i_{\text {mux-l }}$.

$$
\mathbf{H}_{\mathrm{i}}=\mathrm{E}_{\mathrm{i}} \mathbf{H}_{\mathrm{i}-\mathbf{1}}+\mathrm{F}_{\mathrm{i}} .
$$


Example:

$$
\left.\begin{array}{ll}
A_{3} H_{4}+B_{3} H_{3}+C_{3} H_{2}+0+0 & =D_{3} \\
A_{2} H_{3}+B_{2} H_{2}+C_{2} H_{1}+0 & =D_{2} \\
A_{1} H_{2}+B_{1} H_{1}+C_{1} H_{0} & =D_{1}
\end{array}\right\}
$$

Given: $H_{4}, H_{0}$ and $A_{j}, B_{i}, C_{i}$ for $j=1 \rightarrow i=3$.

To calculate $\mathrm{H}_{3}, \mathrm{H}_{2}$, and $\mathrm{H}_{1}$, define the following recursive equations:

$$
\begin{aligned}
& E_{3} H_{2}+F_{3}=H_{3}, \\
& E_{2} H_{1}+F_{2}=H_{2}, \\
& E_{1} H_{0}+F_{1}=H_{1} .
\end{aligned}
$$

Solving Eq. (C-6) in the form of Eq. (C-7) provides the following identifications:

$$
\begin{array}{lll}
E_{3}=-\frac{C_{3}}{B_{3}} & ; & F_{3}=\frac{D_{3}-A_{3} H_{4}}{B_{3}} \\
E_{2}=-\frac{C_{2}}{A_{2} E_{3}+B_{2}} ; & F_{2}=\frac{D_{2}-A_{2} F_{3}}{A_{2} E_{3}+B_{2}} \\
E_{1}=-\frac{C_{1}}{A_{1} E_{2}+B_{1}} \quad ; & F_{1}=\frac{D_{1}-A_{1} F_{2}}{A_{1} E_{2}+B_{1}} .
\end{array}
$$

or:

$$
E_{i}=-\frac{C_{i}}{A_{i} E_{i+1}+B_{i}} \text { and } F_{i}=\frac{D_{i}-A_{i} F_{j+1}}{A_{i} E_{j+1}+B_{i}}
$$

Substitute: $E_{i}$ and $F_{i}$ into $E_{q}$. (C-7).

REC/gW

$-50$. 